\title{
Two-Stage Path Planning Approach for Solving Multiple Spacecraft Reconfiguration Maneuvers
}

\author{
Georges S. Aoude, Jonathan P. How and Ian M. Garcia
}

\begin{abstract}
The paper presents a two-stage approach for designing optimal reconfiguration maneuvers for multiple spacecraft in close proximity. These maneuvers involve well-coordinated and highly-coupled motions of the entire fleet of spacecraft while satisfying an arbitrary number of constraints. This problem is complicated by the nonlinearity of the attitude dynamics, the non-convexity of some of the constraints, and the coupling that exists in some of the constraints between the positions and attitudes of all spacecraft. While there has been significant research to solve for the translation and/or rotation trajectories for the multiple spacecraft reconfiguration problem, the approach presented in this paper is more general and on a larger scale than the problems considered previously. The essential feature of the solution approach is the separation into two stages, the first using a simplified planning approach to obtain a feasible solution, which is then significantly improved using a smoothing stage. The first stage is solved using a bi-directional Rapidly-exploring Random Tree (RRT) planner. Then the second step optimizes the trajectories by solving an optimal control problem using the Gauss pseudospectral method (GPM). Several examples are presented to demonstrate the effectiveness of the approach for designing spacecraft reconfiguration maneuvers.
\end{abstract}

\section{INTRODUCTION}

Formation flying has been extensively investigated as a means to expand the capabilities of space missions focused on obtaining magnetosphere and radiation measurements, gravity field measurements, and 3-D mapping for planetary explorers (to name a few) [1-4]. The use of fleets of small satellites, instead of a single monolithic satellite, would enable longer-baseline/higherresolution imagery and interferometry and yield robust and redundant fault-tolerant spacecraft system architectures, which should improve the science data return [5, 6]. Achieving these benefits will put tight requirements on the high-level mission management and coordination between spacecraft, including the communication, path planning algorithms, and autonomous fault detection and recovery [7-9].

There are several types of trajectory design problems for formation flying spacecraft and two key ones include: 1) reconfiguration, which consists of maneuvering a fleet of spacecraft from one formation to another, and 2) station-keeping, which consists of keeping a cluster

Presented as paper NASA/CP-2007-214158 15-5 at the 20th International Symposium on Space Flight Dynamics in Annapolis, MD, USA, 2007

G. S. Aoude, Ph. D. Candidate, Dept. of Aeronautics and Astronautics, MIT, Cambridge, MA 02139, USA, gaoude@mit.edu

J. P. How, Professor, Dept. of Aeronautics and Astronautics, MIT, Cambridge, MA 02139, USA, jhow@mit.edu

I. G. Garcia, GN\&C engineer, Masten Space Systems, Mojave, CA 93502, USA, igarcia@masten-space.com 


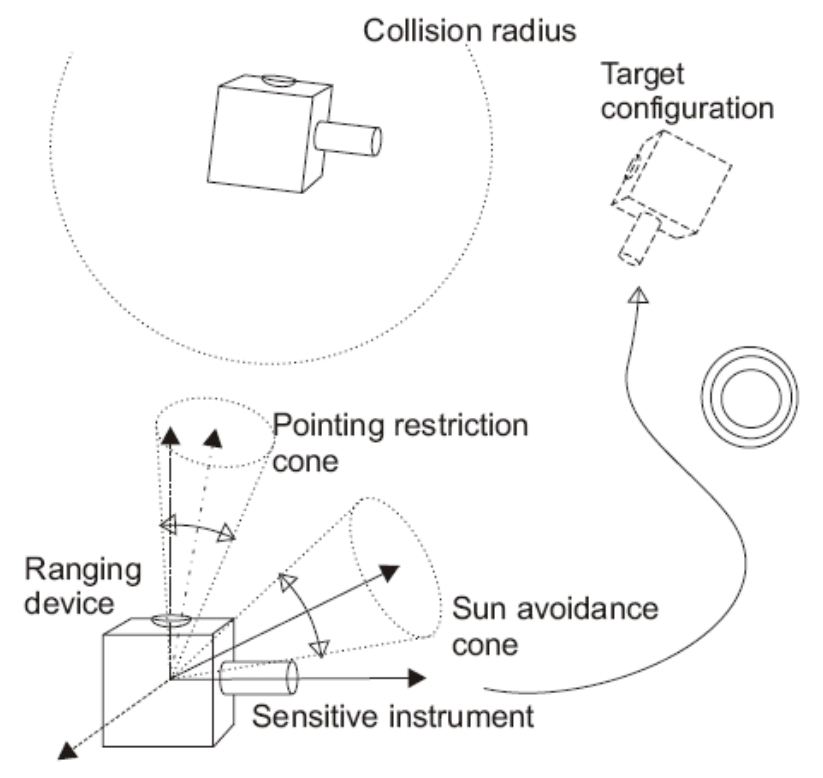

Fig. 1: The formation reconfiguration problem [11]

of fleet of spacecraft in a specific formation for a determined part of the trajectory. Both types of formation flying maneuvers must be addressed for deep-space missions where the relative spacecraft dynamics can typically be reduced to rigid body motion, or planetary orbital environment flying missions where spacecraft are subjected to significant orbital dynamics and environmental disturbances [10].

This paper focuses on the trajectory design of reconfiguration maneuvers of multiple spacecraft in a deep space environment. They consist of moving and rotating a group of $N$ spacecraft from an initial configuration to a desired final configuration, while satisfying different types of constraints (see Figure 1). For vehicles operating in close proximity, these constraints may consist of collision avoidance, restrictions on the region of the sky where certain spacecraft instruments can point (e.g., a sensitive instrument that cannot point at the Sun), or restrictions on pointing towards other spacecraft (e.g., requirements on maintaining inter-spacecraft communication links and having cold science instruments avoid high temperature components on other vehicles). It is also desirable to optimize some performance index (fuel, energy, maneuver time, etc.) [10]. This problem is particularly difficult because of the nonlinearity of the attitude dynamics, the non-convexity of some of the constraints, and the coupling that exists in some of the constraints between the positions and attitudes of all spacecraft. This coupling requires that the optimal translation and attitude planning problems be solved simultaneously as a single $6 N$ DOF problem. This is clearly challenging given that the planning algorithms should eventually be fast enough for an online implementation. 


\section{SURVEY OF PREVIOUS WORK}

The design of constrained and unconstrained translation and attitude spacecraft maneuvers has been the subject of extensive research, but most of this work considers the two problems separately. Refs. [12, 13] proposed the use of Mixed Integer Linear Programming (MILP) or Mixed Integer Linear Matrix Inequalities (MI/LMI) techniques to solve the trajectory design problem. These methods require several simplifications to formulate the problem, such as the use of linearized systems dynamics and constraints in the MILP form [12], and are computationally infeasible for large problems [13]. Potential functions have been widely used [14, 15], but computing a potential function that is free of local minima is computationally very hard for any non-trivial set of constraints [16]. Furthermore, this approach cannot guarantee that the resulting trajectories are collision free, which is critical in spacecraft formation flying missions.

Another popular approach that has been investigated recently with great success in motion planning research is the use of randomized motion planning algorithms such as the probabilistic roadmap (PRM) planners [17, 18], and their incremental counterparts the Rapidly-exploring Random Trees (RRT) algorithms. But the application of RRTs on spacecraft reconfiguration problems was limited to 1) a problem involving a single spacecraft with no pointing constraints [19], 2) a problem considering the attitude maneuver of one spacecraft [16], and 3) a multispacecraft reconfiguration problem that solves for only the translation trajectory [20]. It is only very recently that the more general case of combined translation and attitude reconfiguration of multiple spacecraft problems has been addressed using RRTs [21, 22]. This approach consists of a two-stage planning algorithm, similar to the one developed in this research. However, its second stage, also called the "smoothing" step, is based on linearizing a nonlinear optimization problem around the feasible solution generated by the first stage. This induces linearization errors in the solution of the problem, which can make the results infeasible. This paper extends the approach developed in Ref. [21, 22] by efficiently solving the nonlinear problem instead of linearizing it.

Several researchers have recently explored using pseudospectral methods for nonlinear trajectory optimization problems related to aerospace applications [23-25]. Ref. [26] showed the general applicability of pseudospectral methods to the problem of optimal spacecraft formation flying trajectory design. But to bring pseudospectral methods closer to real-time applications, current research is focusing on reducing their computational burden [27]. Providing an initial feasible solution to the nonlinear optimization solver can significantly decreases the computation times of the pseudospectral methods. The reason is that complex trajectory optimization problems (e.g., multi-spacecraft reconfiguration maneuvers) typically have non-convex feasible regions. These regions increase the difficulty of nonlinear solvers to converge especially if initialized with an arbitrary, and possibly infeasible, initial guess [27]. But ensuring the feasibility of the initial guess for a trajectory optimization problem is complex itself since it requires solving 
a path planning problem with general constraints, which is NP-hard [28]. Ref. [25] suggests a "warm start", which uses the solution of a previous optimization as an initial guess to the current problem, to reduce the overall computation times. This idea is similar to the mesh refinement technique introduced in Ref. [29], which starts with a coarse grid (i.e. low number of discretization nodes), and if necessary, refines the discretization, and then repeats the optimization. To apply such approaches to online path planning, they should be chosen with care to minimize the inefficiency that they can introduce. The technique presented in this paper is a warm start approach that is obtained by quickly solving a simplified version of a trajectory optimization problem using an improved version of bidirectional RRTs [22]. This technique significantly improves the performance of the pseudospectral method used in solving the multi-spacecraft reconfiguration problem.

\section{SOLUTION CONCEPTS}

This section briefly describes the concepts used in the solution process of the two-stage path planning algorithm. The first stage is based on the concept of Rapidly-exploring Random Trees, a randomized sampling-based planning technique [30, 31]. Then the second stage uses pseudospectral methods as a technique for solving an optimal control problem. This two-stage technique extends the original ideas in Ref. [11] to improve the second step by using a specific pseudospectral method, called the Gauss pseudospectral method (GPM) [32] ${ }^{1}$.

\section{Two-Stage Path Planning}

The constraints encountered in spacecraft reconfiguration maneuver problems fall into two main categories, (a) kinematic and (b) dynamic. Kinematic constraints address the motion of the spacecraft under consideration, but ignore the forces behind the motion, which are captured in the dynamic constraints. Path planning for reconfiguration maneuvers is a challenging task even when considering each set of constraints individually [21]. When addressing these two types of constraints simultaneously, the problem is known as kinodynamic motion planning [33], which has been traditionally implemented using two common approaches: "two-stage" planning and "state-space" formulation [33, 34]. Unlike the state-space approach, where the dynamic constraints are taken into account from the start of the algorithm [35, 36], the twostage formulation consists of first finding a feasible path that satisfies the kinematic constraints, and then optimizing this path to include the dynamic constraints [21, 37]. This paper develops a two-stage approach for solving reconfiguration maneuvers of multiple spacecraft. Examples of complex maneuvers including up to five spacecraft illustrate this approach.

\footnotetext{
${ }^{1} \mathrm{GPM}$ is just one of several options that exist. It was primarily chosen because the GPOCS software was readily available.
} 


\section{Rapidly Exploring Random Trees}

The first stage of the two-stage algorithm developed in this paper concentrates on finding any feasible trajectory for the problem, postponing the "smoothing" or cost improvement to the second stage. However, finding a feasible path with guarantees is by itself very difficult because the path planning problem becomes intractable for high dimensional problems like the multiple spacecraft reconfiguration maneuver problem. But it has been shown that if the guaranteed completion is relaxed, larger problems can be solved using randomized path planning algorithms, such as the Probabilistic Roadmaps (PRMs) [17]. Rapidly exploring Random Trees (RRTs), a recent variant of PRMs introduced in Refs. [30, 31], was developed for planning under differential constraints, but it has been applied mostly in ordinary motion planning. The RRT algorithm efficiently explores high-dimensional spaces, therefore it can quickly find a feasible solution even in highly constrained environments.

RRTs have several nice properties [30]. We emphasize two of them: 1) their expansion is heavily biased towards unexplored areas of the configuration space and 2) the RRT algorithm is probabilistically complete i.e., the probability of finding a feasible path approaches one as the number of iterations increases. RRTs and their variants have been applied successfully in several applications in different areas of research including robotics and graphics [38]. This paper uses an improved version of the well known bidirectional RRTs, a technique that has been introduced and shown to be a very fast planner for path planning problems when differential constraints are ignored [22].

\section{Pseudospectral Methods}

The second stage of the planning algorithm developed in this paper is formulated as an optimal control problem with path constraints. Numerical methods for solving this type of problems fall into two general categories: direct methods and indirect methods [39].

In an indirect method, the optimal solution is found by solving a Hamiltonian boundary-value problem derived from the first-order necessary conditions for optimality. The primary advantages of indirect methods are that they exhibit a high accuracy in their solution and that they guarantee that the solution satisfies the first-order optimality conditions [32]. However, indirect methods have several disadvantages including possible difficulties in deriving the Hamiltonian boundaryvalue problem, small radii of convergence, and the requirement of a good initial guess for both the states and costates [39].

In a direct method, the continuous-time optimal control problem is transcribed to a nonlinear programming problem (NLP). Well developed algorithms and softwares are then used to solve numerically the resulting NLP. One of the main advantages of direct methods is that the optimality conditions of the optimal control problem do not need to be derived. Their disadvantage is that, 
depending on the type of direct method, their solution may not contain any costate information or may result in an inaccurate costate [32].

As the number of spacecraft in the reconfiguration problem increases, solving the Hamiltonian boundary value problem becomes increasingly difficult, if not impossible. Moreover, advances in direct methods, such as the pseudospectral methods [23, 40-42], have improved the accuracy of the costate information compared to earlier direct methods.

The states and controls in pseudospectral methods are parameterized using a basis of global polynomials which are derived from an appropriate set of discretization points[40, 42]. The use of global orthogonality makes it simple to transform the original problem into a set of algebraic equations. The discretized optimal control problem is then transcribed to a nonlinear program which can then be solved using an off-the-shelf nonlinear solver. This paper uses the Gauss pseudospectral method (GPM), one of the newest numerical approaches in the literature today, that has shown promise both in the solution and in the post-analysis optimality [32].

\section{PROBLEM FORMULATION}

The general reconfiguration problem resides in finding a trajectory of $N$ spacecraft from time 0 to time $T$. Let $\boldsymbol{p}_{i}(t)$ be a point of the trajectory of a single spacecraft at time $t$. This point consists of

$$
\boldsymbol{p}_{i}(t)=\left[\boldsymbol{x}_{i}^{T}(t), \boldsymbol{u}_{i}^{T}(t)\right]^{T}
$$

where $\boldsymbol{x}_{i}(\mathrm{t})$ and $\boldsymbol{u}_{i}(\mathrm{t})$ represent the state and control inputs at each time $t$, respectively,

$$
\begin{aligned}
\boldsymbol{x}_{i}(t) & =\left[\boldsymbol{r}_{i}^{T}(t), \dot{\boldsymbol{r}}_{i}^{T}(t), \boldsymbol{\sigma}_{i}^{T}(t), \boldsymbol{w}_{i}^{T}(t)\right] \\
\boldsymbol{u}_{i}(t) & =\left[\boldsymbol{f}_{i}^{T}(t), \boldsymbol{\tau}_{i}^{T}(t)\right]
\end{aligned}
$$

and where $\mathrm{i} \in 1 \ldots N$ indicates the spacecraft. $\boldsymbol{r}_{i}(\mathrm{t}) \in \mathbb{R}^{3}$ is the position of its center, $\dot{\boldsymbol{r}}_{i}(\mathrm{t}) \in$ $\mathbb{R}^{3}$ is its velocity, $\boldsymbol{w}_{i}(\mathrm{t}) \in \mathbb{R}^{3}$ its angular velocity, and $\boldsymbol{\sigma}_{i}(\mathrm{t}) \in \mathbb{S O}^{3}$ is its attitude representation in modified Rodrigues parameters (MRP) [43]. The benefits of the choice of MRP over other attitude representations for this spacecraft reconfiguration maneuver problem is explained in Ref. [44]. All these variables are measured with respect to a local inertially fixed frame. $\boldsymbol{f}_{i}(\mathrm{t})$ $\in \mathbb{R}^{3}$ represents the control input force, and $\boldsymbol{\tau}_{i}(\mathrm{t}) \in \mathbb{R}^{3}$ the control input torque. Therefore,

$$
\boldsymbol{p}(t)=\left[\ldots, \boldsymbol{p}_{i}^{T}(t), \ldots\right]^{T}
$$

represents a point in the composite trajectories of all the spacecraft at time $t$. Since the interest of this research is in deep space missions, the translation dynamics are approximated with a 
simple double integrator [45]

$$
\left[\begin{array}{c}
\dot{\boldsymbol{r}}_{i}(t) \\
\ddot{\boldsymbol{r}}_{i}(t)
\end{array}\right]=\left[\begin{array}{cc}
0_{3 \times 3} & I_{3 \times 3} \\
0_{3 \times 3} & 0_{3 \times 3}
\end{array}\right]\left[\begin{array}{c}
\boldsymbol{r}_{i}(t) \\
\dot{\boldsymbol{r}}_{i}(t)
\end{array}\right]+\left[\begin{array}{c}
0_{3 \times 3} \\
\frac{I_{3 \times 3}}{M}
\end{array}\right] \boldsymbol{f}_{i}(t)
$$

where $\mathrm{M} \in \mathbb{R}$ is the mass, assumed to be the same for all spacecraft for simplicity. $I_{3 \times 3}$ is the $3 \times 3$ identity matrix, and $0_{3 \times 3}$ is the $3 \times 3$ zero matrix. But the approach developed in this paper can also be applied to other types of missions (e.g., low Earth orbit) with more complex relative dynamics.

The attitude dynamics in MRP notation of the spacecraft considered as a rigid body are

$$
\begin{aligned}
\dot{\boldsymbol{\sigma}}_{i}(t) & =R\left(\boldsymbol{\sigma}_{i}(t)\right) \boldsymbol{w}_{i}(t) \\
J \dot{\boldsymbol{w}}_{i}(t) & =-\boldsymbol{w}_{i}(t) \times J \boldsymbol{w}_{i}(t)+\boldsymbol{\tau}_{i}(t) \\
& =-S\left(\boldsymbol{w}_{i}(t)\right) J \boldsymbol{w}_{i}(t)+\boldsymbol{\tau}_{i}(t)
\end{aligned}
$$

where $J \in \mathbb{R}^{3}$ is the spacecraft constant inertia matrix, considered to be the same for all spacecraft for simplicity. $S \in \mathbb{R}^{3 \times 3}$ is the skew-symmetric matrix representing the cross product operation

$$
S(\boldsymbol{a}) \triangleq[\boldsymbol{a} \times]=\left[\begin{array}{ccc}
0 & -a_{3} & a_{2} \\
a_{3} & 0 & -a_{1} \\
-a_{2} & a_{1} & 0
\end{array}\right], \quad \forall \boldsymbol{a} \in \mathbb{R}^{3}
$$

The Jacobian matrix $R \in \mathbb{R}^{3 \times 3}$ for MRP attitude representation is given by [43]

$$
R\left(\boldsymbol{\sigma}_{i}\right)=\frac{1}{4}\left[\left(1-\boldsymbol{\sigma}_{i}^{T} \boldsymbol{\sigma}_{i}\right) I_{3 \times 3}+2 S\left(\boldsymbol{\sigma}_{i}\right)+2 \boldsymbol{\sigma}_{i} \boldsymbol{\sigma}_{i}^{T}\right]
$$

The path constraints can be divided into two categories: 1) collision avoidance constraints, and 2) pointing restriction constraints. The collision avoidance category contains the inter-spacecraft collision avoidance constraints, which ensure safe separation between every pair of spacecraft, and are written as

$$
\left\|\boldsymbol{r}_{i}(t)-\boldsymbol{r}_{j}(t)\right\| \geq \boldsymbol{R}_{i j}
$$

for $i, j \in 1 \ldots N, i \neq j$, and $\boldsymbol{R}_{i j}$ is the minimum distance allowed between the centers of spacecraft $i$ and $j$. Collision avoidance also contains the obstacle avoidance constraints, which ensure safe maneuvering of every spacecraft among all obstacles, and are written as

$$
\left\|\boldsymbol{r}_{i}(t)-\boldsymbol{l}_{o}(t)\right\| \geq \boldsymbol{R}_{i o}
$$

for every obstacle $o$, and for $i \in 1 \ldots N . \boldsymbol{l}_{o}$ is the position of the center of obstacle $o$, and $\boldsymbol{R}_{i o}$ is the minimum distance allowed between the centers of spacecraft $i$ and obstacle $o$. 
The pointing restriction category contains four types of constraints: 1) absolute stay outside constraints, 2) absolute stay inside constraints, 3) relative stay outside constraints, and 4) relative stay inside constraints. The absolute stay outside constraints can be written as

$$
\boldsymbol{z}_{k}^{T} \boldsymbol{y}_{k i}(t) \leq \cos \theta_{k i}
$$

for every inertial vector $\boldsymbol{z}_{k}$ and every spacecraft $i$. These constraints ensure that the vectors $\boldsymbol{y}_{k i}$ of spacecraft $i$ remain at an angle greater than $\theta_{k i} \in[0, \pi]$ from their corresponding inertial vector $\boldsymbol{z}_{k}$. The vector $\boldsymbol{y}_{k i}$ of spacecraft $i$ represents the body vector $\boldsymbol{y}_{k i B}$ in the inertial coordinate frame. The transformation of coordinates is given by

$$
\boldsymbol{y}_{k}(t)=\operatorname{Rot}^{-1}(\boldsymbol{\sigma}(\boldsymbol{t})) \boldsymbol{y}_{k B}
$$

where $\operatorname{Rot}(\boldsymbol{\sigma}(t))$ is the rotation matrix representation of the MRP attitude vector $\boldsymbol{\sigma}(t)$, which can be written as [43]

$$
R o t(\boldsymbol{\sigma})=I+\frac{4\left(1-\boldsymbol{\sigma}^{T} \boldsymbol{\sigma}\right)}{\left(1+\boldsymbol{\sigma}^{T} \boldsymbol{\sigma}\right)^{2}} S(\boldsymbol{\sigma})+\frac{8}{\left(1+\boldsymbol{\sigma}^{T} \boldsymbol{\sigma}\right)^{2}} S(\boldsymbol{\sigma})^{2}
$$

where $S$ is the matrix defined in (8). It is assumed that $\boldsymbol{y}_{k B}$ and $\boldsymbol{z}_{k}$ are fixed vectors i.e., independent of time $t$.

The absolute stay inside constraints only change the sign of the inequality of (12). They can be written as

$$
\boldsymbol{z}_{l}^{T} \boldsymbol{y}_{l i}(t) \geq \cos \theta_{l i}
$$

for every inertial vector $\boldsymbol{z}_{l}$ and every spacecraft $i$. These constraints ensure that the vectors $\boldsymbol{y}_{l i}$ of spacecraft $i$ remain at an angle smaller than $\theta_{l} \in[0, \pi]$ from their corresponding inertial vector $\boldsymbol{z}_{l}$. The vector $\boldsymbol{z}_{l i}$ is defined the same way as $\boldsymbol{z}_{k}(13)$.

The inter-spacecraft relative stay outside constraints are given by

$$
\hat{\boldsymbol{r}}_{i j}^{T}(t) \boldsymbol{y}_{m i}(t) \leq \cos \theta_{m i}
$$

for $i, j \in 1 \ldots N, i \neq j$, where

$$
\hat{\boldsymbol{r}}_{i j}(t)=\frac{\boldsymbol{r}_{j}(t)-\boldsymbol{r}_{i}(t)}{\left\|\boldsymbol{r}_{j}(t)-\boldsymbol{r}_{i}(t)\right\|}
$$

represents the unit vector pointing from spacecraft $i$ to spacecraft $j$, and $\boldsymbol{y}_{m i}$ is the same as defined above (13). These constraints ensure that the spacecraft vectors $\boldsymbol{y}_{m i}$ of spacecraft $i$ remain at an angle greater than $\theta_{m i} \in[0, \pi]$ from the vector $\hat{\boldsymbol{r}}_{i j}$. 
The inter-spacecraft relative stay inside can be similarly written as

$$
\hat{\boldsymbol{r}}_{i j}^{T}(t) \boldsymbol{y}_{q i}(t) \geq \cos \theta_{q i}
$$

for $i, j \in 1 \ldots N, i \neq j$, where $\hat{\boldsymbol{r}}_{i j}$ is defined the same way as (17). These constraints ensure that the spacecraft vectors $\boldsymbol{y}_{q i}$ of spacecraft $i$ remain at an angle smaller than $\theta_{q i} \in[0, \pi]$ from the vector $\hat{\boldsymbol{r}}_{i j}$.

The boundary conditions specify the initial and final configuration i.e., state of each spacecraft. They can be written as

$$
\begin{gathered}
\boldsymbol{x}_{i}(0)=\boldsymbol{x}_{i s} \\
\boldsymbol{x}_{i}(T)=\boldsymbol{x}_{i f}
\end{gathered}
$$

where $\boldsymbol{x}_{i s}$ represents the state corresponding to the specified starting condition, and $\boldsymbol{x}_{i f}$ the state corresponding to the specified final condition $\forall i \in 1 \ldots N$.

The state and control vectors are restricted to lie within specified bounds

$$
\begin{aligned}
& \boldsymbol{x}_{\text {min }} \leq \boldsymbol{x}_{i}(t) \leq \boldsymbol{x}_{\max } \\
& \boldsymbol{u}_{\text {min }} \leq \boldsymbol{u}_{i}(t) \leq \boldsymbol{u}_{\text {max }}
\end{aligned}
$$

where the inequality is understood to be component wise. The bounds on the input control vectors are typically due to the limited thrust of each spacecraft. The bounds on the velocity vectors are usually characteristic of safety limits. Finally, the position bounds ensure that the problem space is bounded [46].

Finally, the objective is to minimize the total energy of the formation

$$
\boldsymbol{J}=\sum_{i=1}^{N} \int_{0}^{T}\left\|\boldsymbol{f}_{i}(t)\right\|^{2}+\left\|\boldsymbol{\tau}_{i}(t)\right\|^{2} d t
$$

Minimizing the total energy consumption of a formation of spacecraft is an objective for many space missions [47, 48]. Furthermore, the energy is in general directly related to the fuel consumption. So minimizing energy typically leads to less fuel consumption.

\section{SOLUTION APPROACH}

\section{The RRT First Stage}

The first stage of the RRT-GPM algorithm is based on the improved version of the bidirectional rapidly-exploring random trees (RRT) developed by the authors of this paper [22]. It is reproduced in this section for clarity. The original RRT algorithm was developed by Lavalle [30]. The main contribution of Ref. [22] is changing the function that connects two nodes in the RRT from simple 
and fast to slow but much more effective. The new connection function finds links between the nodes by minimizing a distance function to the target while accounting for the constraints. The examples presented in Ref. [22] demonstrate that this new extension of the bidirectional RRT planner decreases the solution times by approximately two orders of magnitude for the types of problems of interest in this paper, therefore enabling the solution of more complex and larger reconfiguration maneuver problems.

In Algorithm 1, $\boldsymbol{T}_{a}$ and $\boldsymbol{T}_{b}$ represent trees having a composite trajectory point $\boldsymbol{p}$ at each node. $\boldsymbol{T}_{a}$ starts from the initial point and $\boldsymbol{T}_{b}$ starts from the final point of the goal trajectory. At each node, the points $\boldsymbol{p}$ are considered at rest, so the position and attitude are the only information of interest in this algorithm. At each iteration, $\alpha(i)$ generates a random point, and then the point in the tree $\boldsymbol{T}_{a}$ with the minimum distance to the point $\alpha(i)$ is found by calling NEAREST( $\boldsymbol{T}_{a}$, $\alpha(i)$ ). Distance in this context represents a weighted summation of rotation and translation. It can be written as

$$
d\left(\boldsymbol{p}_{1}, \boldsymbol{p}_{2}\right)=\sum_{i=1}^{N}\left\|\boldsymbol{r}_{1, i}-\boldsymbol{r}_{2, i}\right\|+K_{a} \angle\left(\boldsymbol{\sigma}_{1, i}, \boldsymbol{\sigma}_{2, i}\right)
$$

where $\angle\left(\boldsymbol{\sigma}_{1, i}, \boldsymbol{\sigma}_{2, i}\right)$ represents the angle of an eigen-axis rotation between attitude $\boldsymbol{\sigma}_{1, i}$ and $\boldsymbol{\sigma}_{2, i}$ for spacecraft $\mathrm{i}$, and $K_{a}$ is a weight factor that relates the translation distance and rotation angle.

POTENTIAL-CONNECT is an artificial potential function based on a distance metric $d\left(\boldsymbol{p}_{1}, \boldsymbol{p}_{2}\right)$ where the obstacle avoidance, restricted pointing, and other constraints are represented by inequality and equality constraints [22]. So POTENTIAL-CONNECT is a search algorithm that tries to find a sequence of feasible points with a decreasing distance to the target point. This search can be formulated as a nonlinear optimization problem (see Algorithm 2). The solution to this problem can be found using a feasible sequential optimization method, and thus guarantees that the sequence of points represents a valid trajectory.

POTENTIAL-CONNECT tries to connect $\boldsymbol{p}$ to $\boldsymbol{p}_{f}$ by moving in small $d p$ increments. These $d p$ increments are restricted to be smaller in norm than $\epsilon$ to guarantee feasibility between adjacent points of the trajectory. Note that the numerical experiments were done using a custom sequential linear solver that computes the solution of a sequence of linear programs with linearized constraints.

So the solution of the first stage consists of a sequence of points from the initial point $\boldsymbol{p}_{i}$ to the final point $\boldsymbol{p}_{f}$. At each point, the spacecraft are assumed to be at rest, and there exists a direct motion to the next point that is guaranteed to satisfy all the constraints. This improved bidirectional RRT planner has been demonstrated to be significantly faster than other similar spacecraft reconfiguration maneuver planners. References [11,22] provide a more detailed analysis of Algorithm 1 and Algorithm 2. 

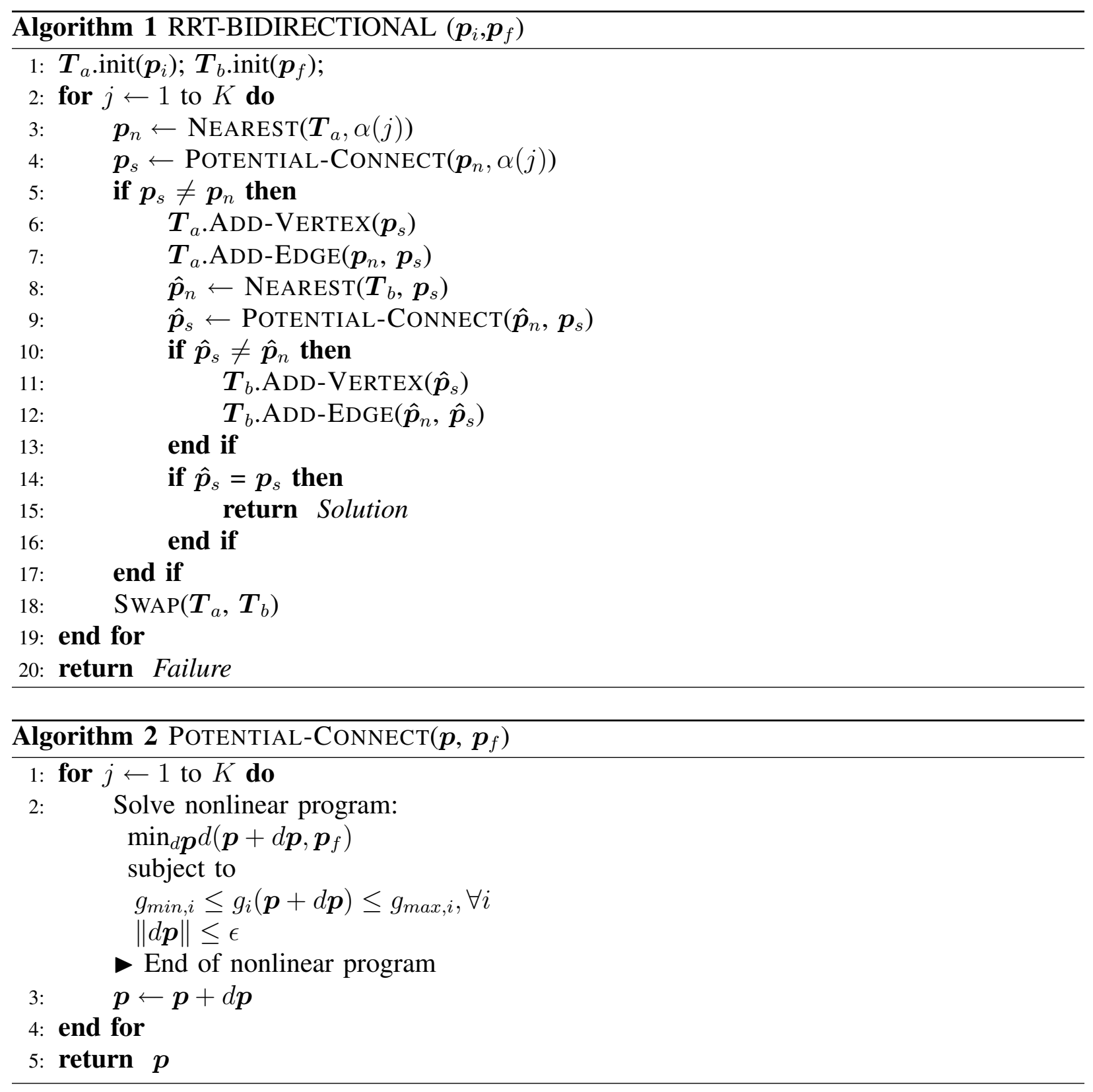

The Augmentation with Feasible Dynamics

A major simplification in the first stage of the RRT-GPM algorithm is based on ignoring the differential constraints of the spacecraft reconfiguration problem. This simplification is made to decrease the computation time of the first stage. The RRT solution of the first stage is suboptimal since the spacecraft are assumed to be at rest at each of the nodes, and no cost function is actually optimized. Therefore, a second stage is needed to improve the cost of the trajectory. A transition step that augments the RRT solution with feasible dynamics is thus required to allow using this 


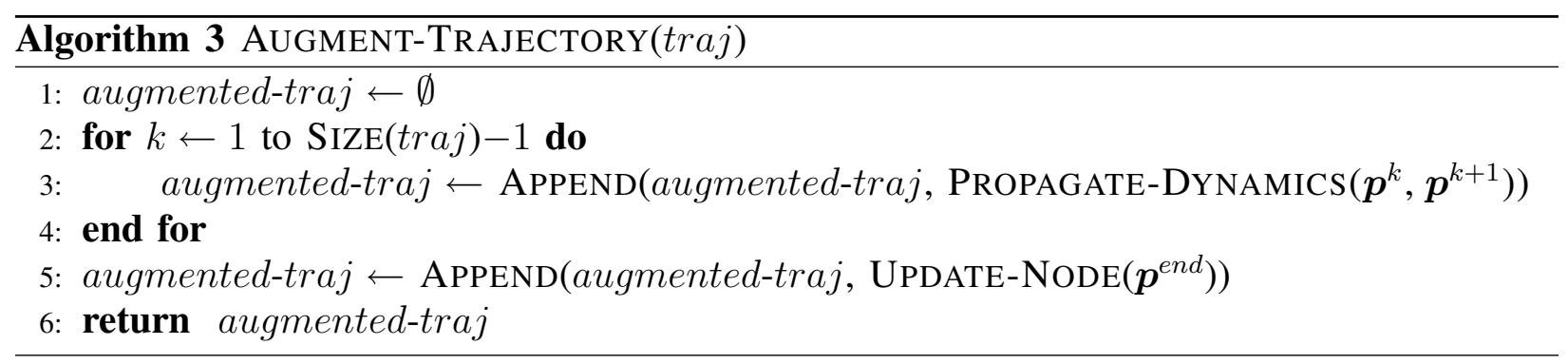

solution as a feasible initial guess to the second stage. So a main requirement of this transition step is to ensure that feasibility is maintained between the first and second stage.

The idea of this transition step starts by adding an intermediate node $p^{\text {inter }}$ half way between each pair of nodes. First assume that the problem consists of only one spacecraft. To propagate the dynamics to $\boldsymbol{p}^{\text {inter }}$, the spacecraft is assumed to accelerate under a constant input force $\hat{f}$ and a constant input torque $\hat{\tau}$. $\hat{f}$ and $\hat{\tau}$ are chosen such that they satisfy the bounds on the forces and torques defined in (22). Once the spacecraft reaches $\boldsymbol{p}^{\text {current }}$, it decelerates under a constant force $-\hat{f}$ and a constant torque $-\hat{\tau}$ until it stops at the next node $p^{\text {next }}$. This is simple way to guarantee that the controls of the spacecraft are satisfied along each consecutive nodes. The smaller the magnitudes of $\hat{f}$ and $\hat{\tau}$ are, the longer the total maneuver time is. Therefore, these values should be chosen to also satisfy the design specifications (e.g., total maneuver time) of the reconfiguration maneuver. Note that the restriction that the intermediate node lies exactly in between the original pair of nodes only exists in the initial guess. After the initial guess is given to the GPM stage, that restriction disappears, along with the assumption of fixed forces and torques.

To expand this idea to multiple spacecraft, a constraint is added to ensure that that all spacecraft reach the intermediate node $\boldsymbol{p}^{\text {inter }}$ at the same instant of time. This time synchronization is a simple way to ensure the feasibility of the algorithm when considering multiple spacecraft. But again, this constraint is relaxed after the guess is given to the second stage, i.e., the final solution of the RRT-GPM approach is not required to satisfy it. First of all, $t_{\max }$ is computed. $t_{\max }$ represents the maximum time needed by all spacecraft to reach $\boldsymbol{p}^{\text {inter }}$, if they all move under the same constant force $\hat{f}$ and rotate under the same constant torque $\hat{\tau}$. Then fixing the time to reach $\boldsymbol{p}^{\text {inter }}$ to be $t_{\max }$ for all spacecraft, the constant forces and torques responsible to move each spacecraft are recomputed. Therefore some spacecraft will be designed to move under forces smaller than $\hat{f}$, and torques smaller than $\hat{\tau}$. Algorithm 3 contains the main steps of the transition algorithm. traj is the RRT output of the first stage, and $\boldsymbol{p}^{k}$ represents a point in the composite trajectories of all the spacecraft at node $k$. To make the algorithms simpler, it is assumed that the unknown values of the RRT output i.e., velocities, forces and torques, are 

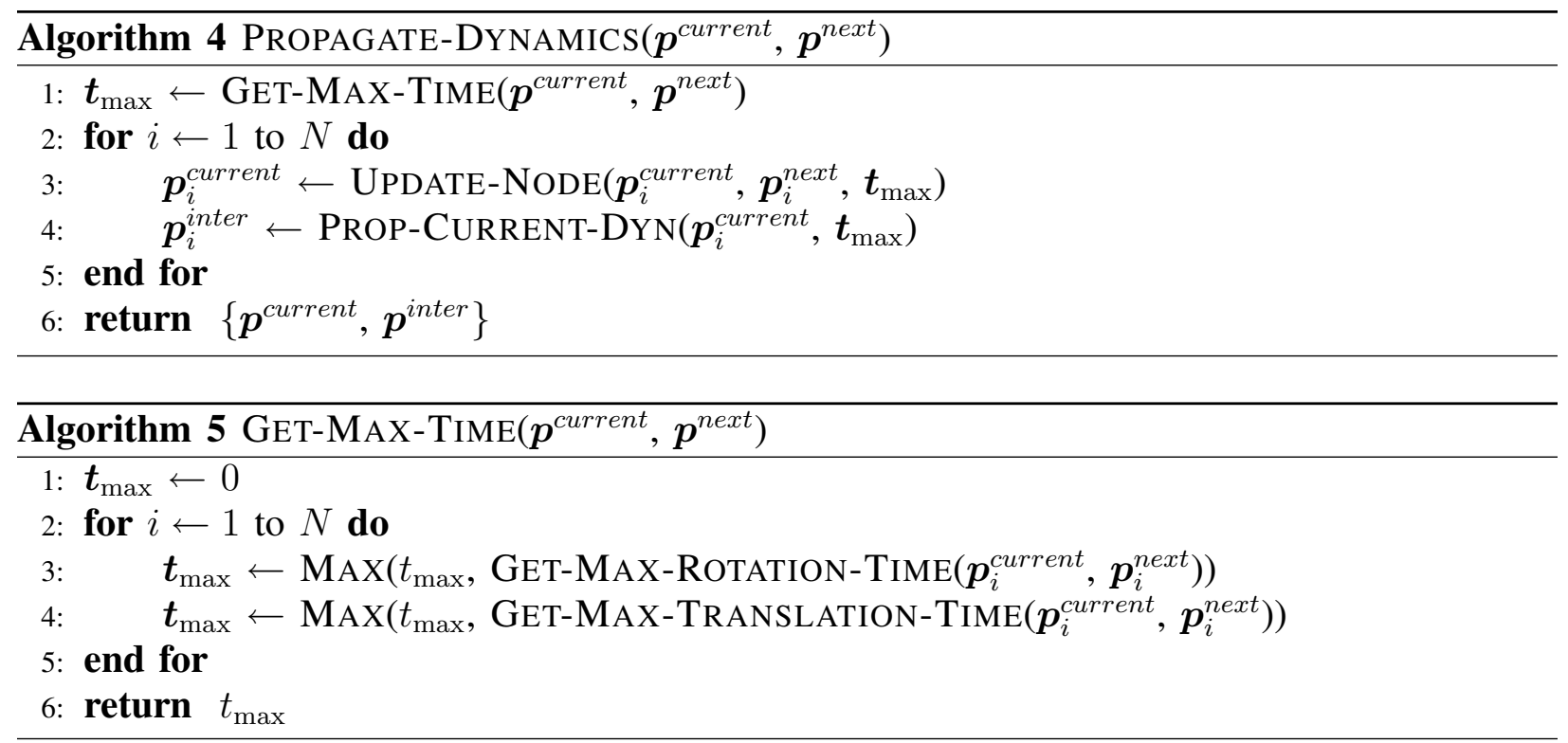

all set to zero by default. An UPDATE operation sets these variables to the correct values, and a PROPAGATE operation creates new nodes using information of existing updated nodes. Lines 2-4 in Algorithm 3 propagates the dynamics between each consecutive pairs of nodes of the trajectory. Line 5 ensures that the dynamics of the last node of the trajectory are also updated. Algorithm 4 describes how the dynamics are propagated between each pair of nodes. Line 1 calls Get-MAX-Time to compute $t_{\max }$. Refer to Algorithm 5 for the steps involved in GETMAX-Time. Continuing with Algorithm 4, line 3 updates the forces and torques of the current node $\boldsymbol{p}^{\text {current }}$, which are ensured to be feasible by construction. The velocities at $\boldsymbol{p}^{\text {current }}$ remain zeros to satisfy the assumption of the first stage algorithm. Line 4 propagates the dynamics to the intermediate node $\boldsymbol{p}^{\text {inter }}$ by using the logic explained earlier in this section.

Note that the transition step has to also ensure that the velocities of the spacecraft always lie between the permissible bounds. This check can be incorporated easily in the GET-MAX-TIME function, but it is not usually a dominating factor. The reason is that the main limitation of the spacecraft is typically the maximum thrust it can exert, not the maximum velocity it is allowed to reach. The transition step runs in $O(N n)$, where $N$ is the size of the formation, and $n$ is the total number of nodes in the RRT output.

In summary, the transition step is a technique that augments the output of the RRT output of the first stage of the RRT-GPM algorithm with feasible dynamics. It consists of adding intermediate nodes with a complete specific set of feasible dynamics, and synchronizes all spacecraft to get to each node at the same time with feasible forces and torques. The whole process can be easily automated. 


\section{The GPM Second Stage}

The second stage of the RRT-GPM algorithm formulates the multiple spacecraft reconfiguration maneuver as an optimal control problem. This optimal control problem is discretized at some specific discretization points called the Legendre-Gauss (LG) points, and then transcribed into a nonlinear program (NLP) by approximating the states and controls using Lagrange interpolating polynomials. The resulting NLP is then solved using an off-the-shelf nonlinear solver [49]. The augmented RRT output of the first stage is used as initial guess in solving the NLP, and is essential in 1) reducing the computation times of the solution process and 2) solving more complex reconfiguration problems.

Pseudospectral methods have been a popular choice among numerical direct methods to solve optimal control problem due to their ability to provide accurate solutions of the costates without requiring the use of analytical differential equations of the adjoints [42]. Another important feature of the pseudospectral methods is that they typically have faster convergence rate than other direct methods. They are also known to demonstrate a "spectral accuracy" [50].

This paper uses the Gauss pseudospectral method (GPM) for which the Karush-Kuhn-Tucker (KKT) conditions of the NLP have been shown to be exactly equivalent to the discretized form of the first-order optimality conditions of the Hamiltonian boundary value problem (HBVP) [27]. Therefore a solution to the NLP is guaranteed to satisfy the optimality conditions traditionally used in indirect methods, thus removing a primary disadvantage of direct methods. Ref. [27, $32,51]$ describe the pseudospectral methods, and more specifically for the Gauss pseudospectral method.

The general formulation adopted in this second stage is the following [32, 52]. Determine the state, $\boldsymbol{x}(\mathrm{t})$, and control, $\boldsymbol{u}(\mathrm{t})$, that minimize the cost functional

$$
J=\Phi\left(\boldsymbol{x}\left(t_{0}\right), t_{0}, \boldsymbol{x}\left(t_{f}\right), t_{f}\right)+\int_{t_{0}}^{t_{f}} g(\boldsymbol{x}(t), \boldsymbol{u}(t), t) d t
$$

subject to the dynamic constraints

$$
\dot{\boldsymbol{x}}=\boldsymbol{F}(\boldsymbol{x}(t), \boldsymbol{u}(t), t) \in \mathbb{R}^{n}
$$

the boundary condition

$$
\boldsymbol{\phi}\left(\boldsymbol{x}\left(t_{0}\right), t_{0}, \boldsymbol{x}\left(t_{f}\right), t_{f}\right)=\mathbf{0} \in \mathbb{R}^{q}
$$

the inequality path constraints

$$
\boldsymbol{C}(\boldsymbol{x}(t), \boldsymbol{u}(t), t) \leq \mathbf{0} \in \mathbb{R}^{c}
$$

where $t_{0}$ is the initial time, $t_{f}$ is the final time, and $t \in\left[t_{0}, t_{f}\right]$. 
The optimal control problem of equations (25)-(28) is referred as the continuous Bolza problem. This problem is defined on $\left[t_{0}, t_{f}\right]$, where $t_{0}$ and $t_{f}$ can be free or fixed variables. However, the Gauss pseudospectral method used to solve this problem requires a fixed time interval, such as $[-1,1]$. The mapping between the time interval $t \in\left[t_{0}, t_{f}\right]$ and the time interval $\varsigma \in[-1,1]$ can be written as

$$
\varsigma=\frac{2 t}{t_{f}-t_{0}}-\frac{t_{f}+t_{0}}{t_{f}-t_{0}}
$$

Rewrite the optimal control problem as

$$
J=\Phi\left(\boldsymbol{x}(-1), t_{0}, \boldsymbol{x}(1), t_{f}\right)+\frac{t_{f}-t_{0}}{2} \int_{-1}^{1} g\left(\boldsymbol{x}(\varsigma), \boldsymbol{u}(\varsigma), \varsigma ; t_{0}, t_{f}\right) d \varsigma
$$

subject to the constraints

$$
\begin{array}{r}
\dot{\boldsymbol{x}}=\frac{t_{f}-t_{0}}{2} \boldsymbol{F}\left(\boldsymbol{x}(\varsigma), \boldsymbol{u}(\varsigma), \varsigma ; t_{0}, t_{f}\right) \in \mathbb{R}^{n} \\
\boldsymbol{\phi}\left(\boldsymbol{x}(-1), t_{0}, \boldsymbol{x}(1), t_{f}\right)=\mathbf{0} \in \mathbb{R}^{q} \\
\boldsymbol{C}\left(\boldsymbol{x}(\varsigma), \boldsymbol{u}(\varsigma), \varsigma ; t_{0}, t_{f}\right) \leq \mathbf{0} \in \mathbb{R}^{c}
\end{array}
$$

where $\varsigma \in[-1,1]$. (30)-(33) is called the transformed continuous Bolza problem.

In the GPM, the set of $\mathcal{N}$ discretization points includes $\mathcal{K}=\mathcal{N}-2$ interior LG points, the initial point $\varsigma_{0} \equiv-1$, and the final point $\varsigma_{f} \equiv 1$. GPM approximates the states by using a basis of $\mathcal{K}+1$ Lagrange interpolating polynomials, $\mathcal{L}_{i}, i=0 \ldots \mathcal{K}$,

$$
\boldsymbol{x}(\varsigma) \approx \boldsymbol{X}(\varsigma)=\sum_{i=0}^{\mathcal{K}} \boldsymbol{X}\left(\varsigma_{i}\right) \mathcal{L}_{i}(\varsigma)
$$

where

$$
\mathcal{L}_{i}(\varsigma)=\prod_{j=0, j \neq i}^{\mathcal{K}} \frac{\varsigma-\varsigma_{i}}{\varsigma_{i}-\varsigma_{j}}
$$

The control is approximated using a basis of $\mathcal{K}$ Lagrange interpolating polynomials $\mathcal{L}_{i}^{\dagger}, i=$ $1 \ldots \mathcal{K}$

$$
\boldsymbol{u}(\varsigma) \approx \boldsymbol{U}(\varsigma)=\sum_{i=1}^{\mathcal{K}} \boldsymbol{U}\left(\varsigma_{i}\right) \mathcal{L}_{i}^{\dagger}(\varsigma)
$$


where

$$
\mathcal{L}_{i}^{\dagger}(\varsigma)=\prod_{j=1, j \neq i}^{\mathcal{K}} \frac{\varsigma-\varsigma_{i}}{\varsigma_{i}-\varsigma_{j}}
$$

The dynamic constraints are transcribed into algebraic constraints as follows

$$
\sum_{i=0}^{\mathcal{K}} D_{k i} \boldsymbol{X}_{\varsigma_{i}}-\frac{t_{f}-t_{0}}{2} \boldsymbol{F}\left(\boldsymbol{X}\left(\varsigma_{k}\right), \boldsymbol{U}\left(\varsigma_{k}\right), \varsigma_{k} ; t_{0}, t_{f}\right)=\mathbf{0}
$$

where $k=1 \ldots \mathcal{K}$, and $D$ is an $\mathcal{K} \times(\mathcal{K}+1)$ differential approximation matrix, consisting of the derivative of each Lagrange polynomial corresponding to the state at each LG point. This matrix can be computed offline as follows:

$$
D_{k i}=\dot{\mathcal{L}}_{i}\left(\varsigma_{k}\right)=\sum_{l=0}^{\mathcal{K}} \frac{\prod_{j=0, j \neq i, l}^{\mathcal{K}}\left(\varsigma_{k}-\varsigma_{j}\right)}{\prod_{j=0, j \neq i}^{\mathcal{K}}\left(\varsigma_{i}-\varsigma_{j}\right)}
$$

where $k=1 \ldots \mathcal{K}$ and $i=0 \ldots \mathcal{K}$. Note that the collocation of the dynamic constraint only happens at the LG points and not at the boundary points. Two additional variables, $\boldsymbol{X}_{0}$ and $\boldsymbol{X}_{f}$ are defined in this discretization. $\boldsymbol{X}_{0} \equiv \boldsymbol{X}(-1)$, and $\boldsymbol{X}_{f}$ is defined via a Gauss quadrature

$$
\boldsymbol{X}_{f} \equiv \boldsymbol{X}_{0}+\frac{t_{f}-t_{0}}{2} \sum_{k=1}^{\mathcal{K}} w_{k} \boldsymbol{F}\left(\boldsymbol{X}\left(\varsigma_{k}\right), \boldsymbol{U}\left(\varsigma_{k}\right), \varsigma_{k} ; t_{0}, t_{f}\right)
$$

where $w_{k}$ are the Gauss weights. Continuing with the transcription process, (30) is approximated using a Gauss quadrature

$$
J=\Phi\left(\boldsymbol{X}_{0}, t_{0}, \boldsymbol{X}_{f}, t_{f}\right)+\frac{t_{f}-t_{0}}{2} \sum_{k=1}^{\mathcal{K}} w_{k} g\left(\boldsymbol{X}\left(\varsigma_{k}\right), \boldsymbol{U}\left(\varsigma_{k}\right), \varsigma_{k} ; t_{0}, t_{f}\right)
$$

The boundary constraint is written as

$$
\phi\left(\boldsymbol{X}_{0}, t_{0}, \boldsymbol{X}_{f}, t_{f}\right)=\mathbf{0}
$$

Finally, the path constraint is computed at the LG points as

$$
\boldsymbol{C}\left(\boldsymbol{X}\left(\varsigma_{k}\right), \boldsymbol{U}\left(\varsigma_{k}\right), \varsigma_{k} ; t_{0}, t_{f}\right) \leq \mathbf{0}
$$

where $k=1 \ldots \mathcal{K}$. Equations (38), (40), (41), (42) and (43) form an NLP that is the transcription of the modified continuous Bolza problem (MCBP). The solution of the NLP is an approximate solution to the MCBP. 


\section{EXAMPLES}

In this section, examples of different complexity are solved using the RRT-GPM technique described in section SOLUTION APPROACH. In the figures illustrating the examples, the trajectories are shown in solid lines, and each dot represents a time step. The spacecraft are shown on the trajectory every second to fifth time step, depending on the example. The plot axes represent the axes of the local inertially fixed frame. The vectors attached on each spacecraft are the $X, Y$, and $Z$ body axes. Some examples include some fixed obstacles, shown as green sphere-shaped objects. Furthermore, examples that have "stay outside" constraints show red "umbrellas", with a handle showing the direction of the restricted pointing, and a cone of rays illustrating the angle of the constraint. The characteristics of the spacecraft are similar to those of SPHERES microsatellites that were developed in the Space Systems Laboratory at MIT, and the dimensions of the test environment are similar to those of the SPHERES testbed on the International Space Station (ISS) [53-55]. Note that some of the examples are motivated by reconfiguration maneuvers described in Ref. [11].

\section{Implementation Details}

The RRT first stage and the transition step are programmed in $\mathrm{C}++$, and they are compiled in Microsoft Visual Studio .NET 2003. The RRT first stage uses a linear solver based on the GLPK library. The GPM second stage is programmed in Matlab, and uses the GPOCS software package [56]. GPOCS is a MATLAB implementation of the Gauss pseudospectral method for solving optimal control problems. GPOCS relies on SNOPT [49], an SQP solver for large-scale constrained optimization, to solve the NLP formed by the GPM method. The experiments are run on a Pentium 4, $2.2 \mathrm{GHz}$ processor equipped with $1 \mathrm{~GB}$ of RAM. Both optimality and feasibility tolerances are set to $10^{-4}$ in GPOCS. The weighting factor $K_{a}$ introduced in (24) is set to 6 . The number of discretization points used in the second stage is $\mathcal{N}=25$.

\section{Example: Two-Spacecraft Maneuver with Obstacle and Sun Avoidance}

The first example is a two-satellite maneuver that includes obstacle avoidance and sun avoidance constraint. Spacecraft 1 and 2 are initially positioned at $[0,0,0]^{T}$ and $[1,1,1]^{T}$. The maneuver consists of spacecraft 1 and 2 switching positions, and rotating $90^{\circ}$ about the inertial Z-axis, while avoiding pointing at the sun and colliding with a fixed obstacle. The unit vector pointing at the sun is the vector $\left[\frac{1}{\sqrt{2}}, \frac{1}{\sqrt{2}}, 0\right]^{T}$, surrounded by a cone of $25^{\circ}$ half angle. The spacecraft must maintain its "sensitive" instrument (e.g., telescope lens), which is mounted in the direction of the body $\mathrm{X}$ axis, out of the cone. The fixed obstacle is a sphere centered at $[0.3,0.3,0.3]^{T}$ with a radius of $0.15 \mathrm{~m}$. The computation times are $2 \mathrm{sec}$ for the first stage, and $65 \mathrm{sec}$ for 


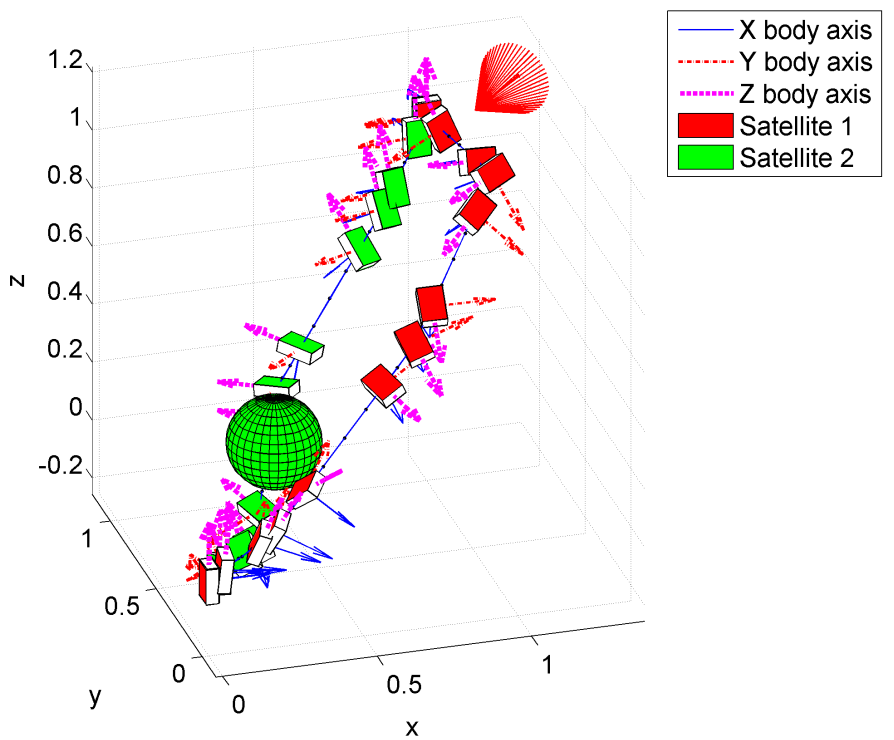

Fig. 2: Example: Two-Spacecraft Maneuver with Obstacle and Sun Avoidance. RRT Output. Spacecraft 1 and 2 switch positions while avoiding colliding with a fixed obstacle and pointing to the sun.

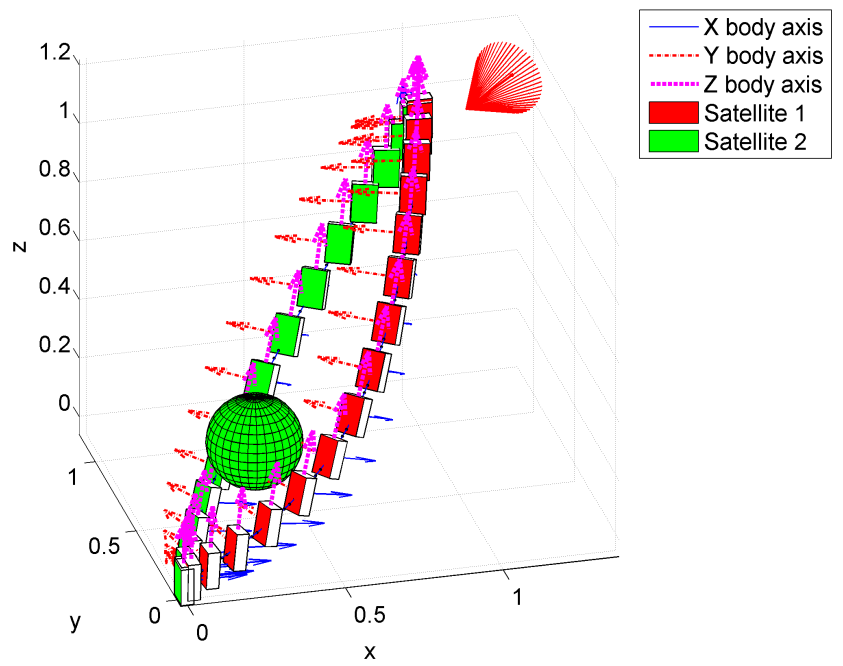

Fig. 3: Final trajectory of the two-spacecraft maneuver with obstacle and sun avoidance example.

the second stage. Figure 2 shows the output of first stage. The trajectory of both spacecraft are feasible, i.e., the spacecraft avoid colliding with the fixed obstacle, and do not point in the sun cone. However, the trajectories are suboptimal. Figure 3 shows the final trajectory, after the RRT output is smoothed through the second stage. The spacecraft follow a trajectory that satisfies all the constraints, and minimizes energy consumption. 


\section{Example: Coupled Two-Spacecraft Maneuver}

This example is a more complex two-spacecraft reconfiguration maneuver. Its complexity is due to the inclusion of inter-spacecraft pointing constraints, which adds coupling between the position and attitude states of the spacecraft. In this example, spacecraft 1 and 2 are initially positioned at $[0,0,0]^{T}$ and $[1,1,1]^{T}$. The maneuver consists of the spacecraft switching positions and rotating $180^{\circ}$ about the inertial vector $\left[\frac{-1}{\sqrt{3}}, \frac{-1}{\sqrt{3}}, \frac{1}{\sqrt{3}}\right]^{T}$, while satisfying an inter-spacecraft pointing constraint and avoiding collisions with the two fixed obstacles. Note that both spacecraft must point their body $\mathrm{X}$ axis (solid blue) to the other spacecraft to within $32^{\circ}$. The centers of the obstacles are located at $[0.5,0.7,0.5]^{T}$ and $[0.5,0.1,0.5]^{T}$, and have a radius of $0.15 \mathrm{~m}$. The computation times are $4 \mathrm{sec}$ for the first stage, and $112 \mathrm{sec}$ for the second stage.

Figure 4 shows the trajectory output after the RRT stage is completed. Notice that both spacecraft maintain their relative pointing, and avoid colliding with the obstacles. Spacecraft 1 passes under the obstacle shown on the right of the figure, and Spacecraft 2 goes over the obstacle shown on the left. However, this strategy is clearly suboptimal, since there is enough space for both spacecraft to go in between the obstacles, and thus reduce fuel consumption. Figure 5 shows the final trajectory produced by the second stage of RRT-GPM path planner. The trajectory of both spacecraft were moved towards the diagonal path, while maintaining feasibility of the constraints.

This maneuver is designed to have a narrow passage in between the obstacles, which is usually hard for path planners to find [57]. Figure 7a shows the inter-spacecraft collision avoidance constraint which is active for a large part of the maneuver, when the spacecraft enter the narrow passage between the obstacles. Figures $6 \mathrm{a}$ and $6 \mathrm{~b}$ show the distance between the centers of spacecraft and the centers of each obstacle. The dashed lines show the minimum permissible distances. The figures illustrate how the spacecraft have to maneuver around the obstacles while avoiding colliding with them. Figure $7 \mathrm{~b}$ shows the inter-spacecraft pointing constraints. Spacecraft 1 and 2 must keep their instrument, which is mounted on their body $\mathrm{X}$ axis, pointing at the other spacecraft to within $32^{\circ}$. Figure $7 \mathrm{~b}$ shows that this is a restrictive constraint since it forces the instrument to be pointing within a thin cone during the entire maneuver. The interspacecraft pointing constraints always stay feasible except around $t=40 \mathrm{~s}$ and $t=80 \mathrm{~s}$, but the deviations are within the feasibility tolerance specified in the GPOCS solver, and are thus acceptable. Finally, it is important to note that the first RRT stage is essential in enabling a solution to coupled maneuver problems similar to this example. The GPOCS solver failed to solve this problem every time it was not initialized with the RRT guess of the first stage. 


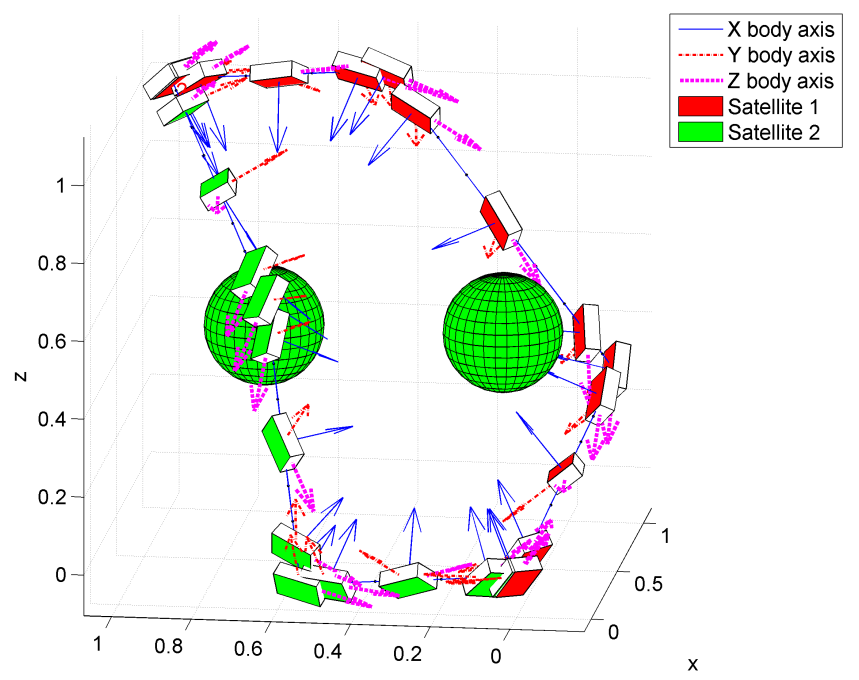

Fig. 4: Example: Two-Spacecraft Maneuver with Inter-Spacecraft Pointing and Obstacle Avoidance. RRT Output. Spacecraft 1 and 2 switch positions while avoiding colliding with two fixed obstacles and keep pointing to each other within $32^{\circ}$.

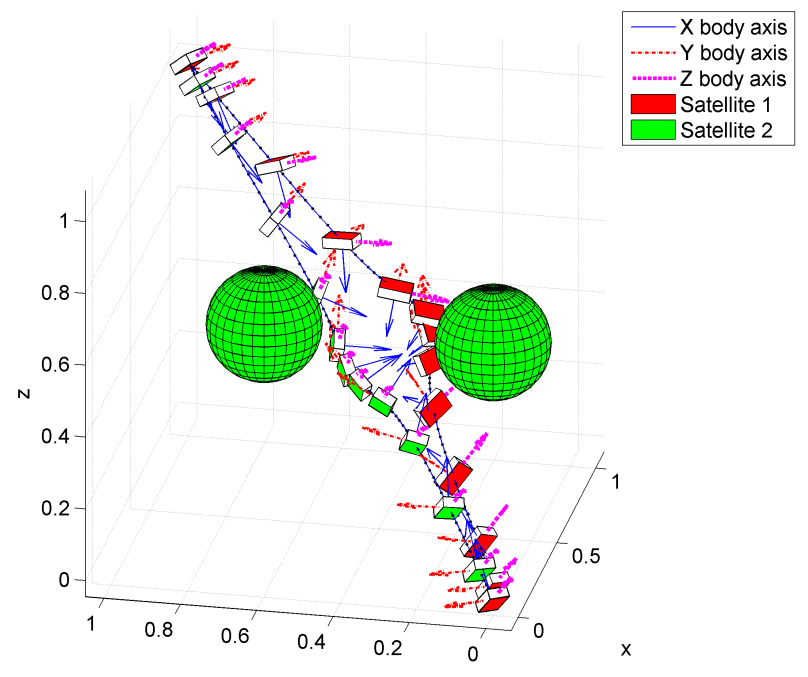

Fig. 5: Final trajectory of the two-spacecraft maneuver with obstacle and inter-spacecraft pointing.

\section{Example: Four-Spacecraft Maneuver}

This example is a more challenging highly coupled reconfiguration maneuver involving four spacecraft with an absolute pointing constraint and several inter-spacecraft constraints. Spacecraft 1 and 2 switch their positions and attitude while pointing their body $\mathrm{X}$ axis to each other within $33^{\circ}$. Spacecraft 1 and 2 are initially located at $[0,0.7,0]^{T}$ and $[0,0,0]^{T}$. Two other spacecraft 3 and 4 are "health-monitoring" spacecraft 1 and 2. Both spacecraft 3 and 4 must end at their respective starting position, $[0,-0.5 .0]^{T}$ and $[0,1.2,0]^{T}$. They also have to keep pointing their 


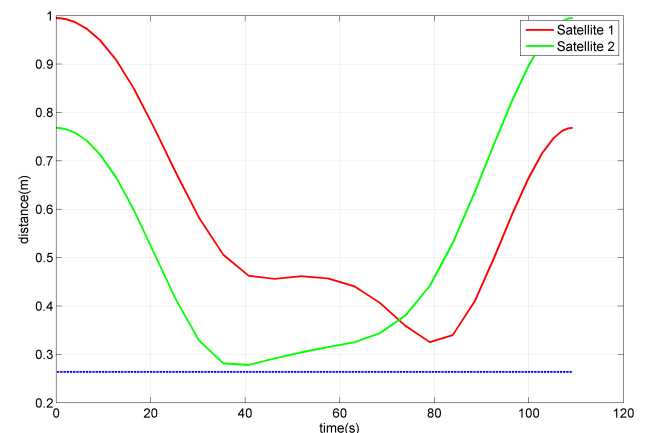

a. Distances between centers of the spacecraft and center of obstacle located at $(0.5,0.7,0.5)$. The dashed line shows the minimum permissible distance.

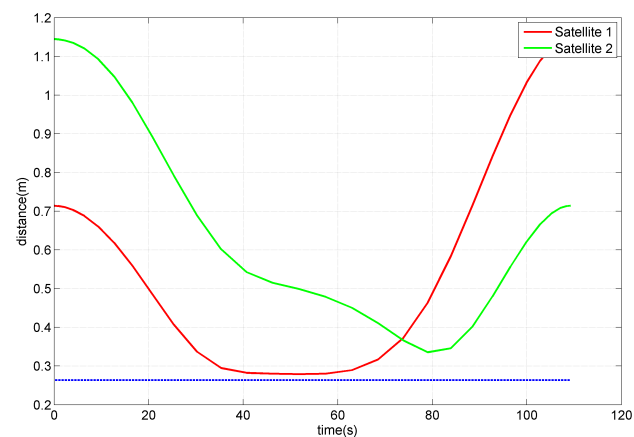

b. Distances between centers of the spacecraft and center of obstacle located at $(0.5,0.1,0.5)$. The dashed line shows the minimum permissible distance.

Fig. 6: Post-analysis of obstacle avoidance constraints for the coupled two-spacecraft maneuver.

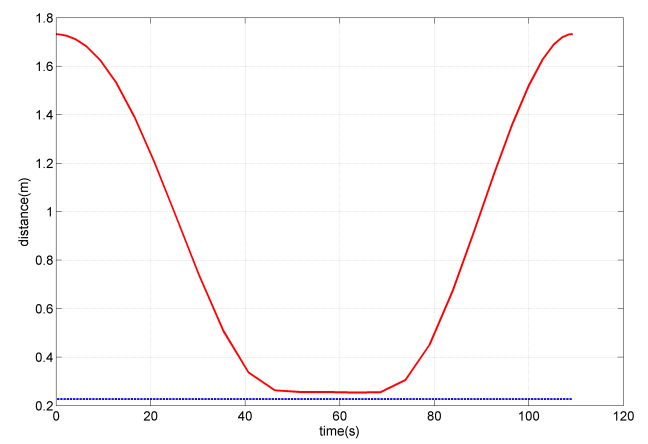

a. Distance between the centers of the spacecraft. The dashed line shows the minimum permissible distance.

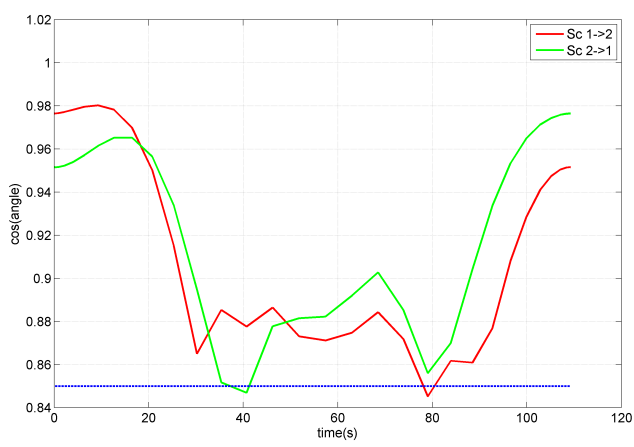

b. Cosines of angles between ranging device vector and relative position of both spacecraft. The dashed line represents the cosine of the maximum angle allowed between the ranging device vector and each of the relative position vectors.

Fig. 7: Post-analysis of inter-spacecraft constraints for the coupled two-spacecraft maneuver.

body $\mathrm{X}$ axis at both spacecraft 1 and 2 to within $30^{\circ}$. All four spacecraft must also avoid pointing their $\mathrm{X}$ body axis in the sun cone. The sun cone is represented by the vector $[1,0,0]^{T}$ pointing at the sun and surrounded by a $20^{\circ}$ half angle cone. The computation times are $17 \mathrm{sec}$ for the first stage, and $302 \mathrm{sec}$ for the second stage.

The trajectory produced by the RRT first stage is shown in Figure 8. Notice that spacecraft 1 and 2 have to leave the X-Y plane in order to keep pointing to each other and avoid pointing in the sun direction. Consequently, spacecraft 3 and 4 have to leave the X-Y plane in order to keep both spacecraft 1 and 2 inside their respective pointing cones. This shows a clear coupling 


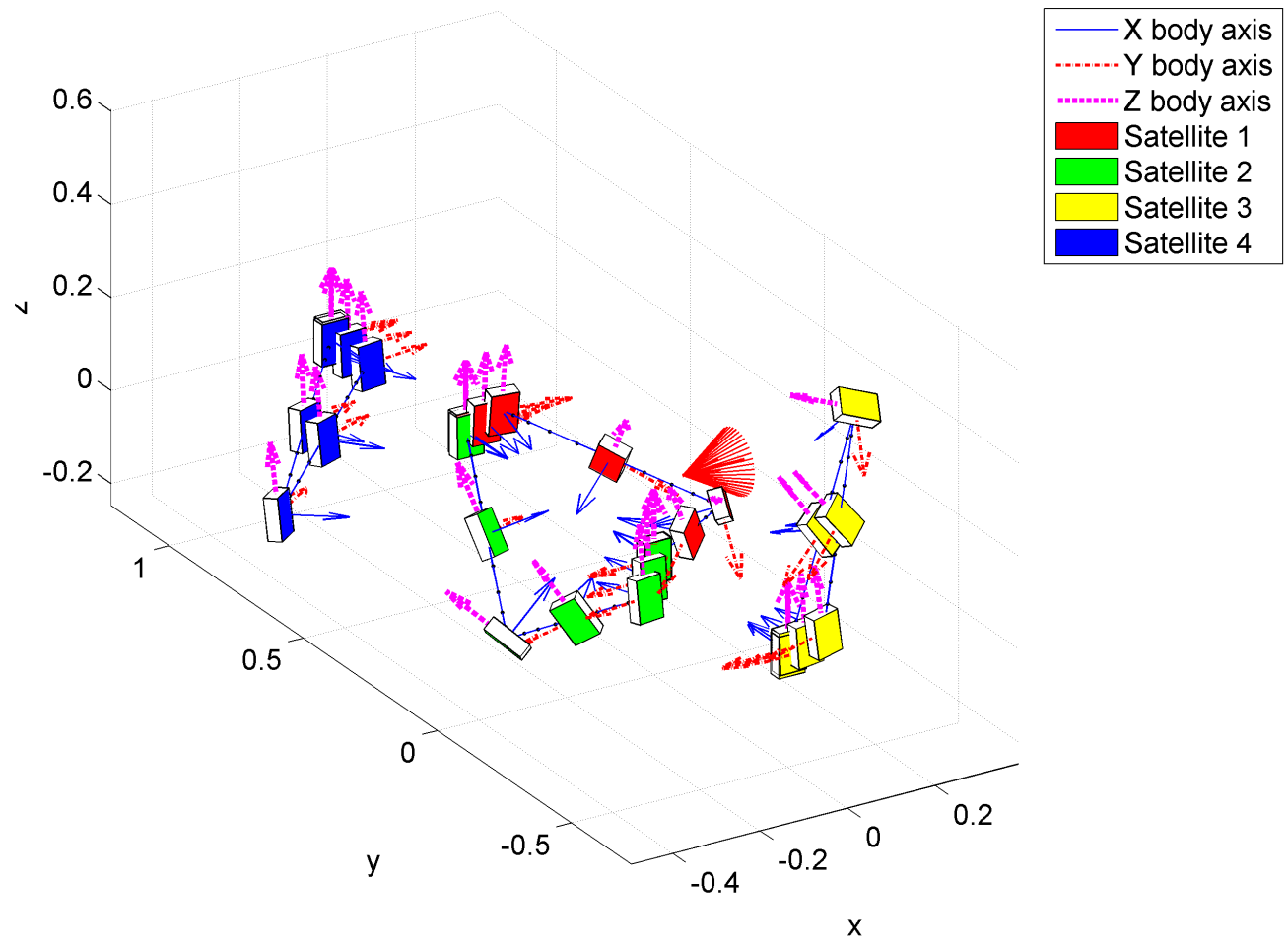

Fig. 8: Example: Four-Spacecraft Maneuver with Inter-Spacecraft Pointing and Absolute Pointing. RRT Output. Spacecraft 1 and 2 switch positions while keep pointing to each other within $33^{\circ}$. Spacecraft 3 and 4 keep both spacecraft 1 and 2 in their specified cone of $30^{\circ}$.

between the positions and attitudes of the spacecraft which is due to the absolute pointing and relative pointing constraints. All spacecraft also avoid colliding with each other. Figure 9 shows the final trajectory produced by the RRT-GPM planner. It is a smoothed version of Figure 8. Figure 9 shows that all of constraints are met. Again, GPOCS failed to solve this problem when the RRT guess of the first stage was not given to it as an initial guess. This shows the importance of the first stage in enabling a solution for complex reconfiguration problems that include coupled constraints.

The following section underlines the importance of initializing GPM methods, and more generally pseudospectral methods, with an initial feasible guess. It reviews the different initialization techniques that have been used for optimal control problems. Then it introduces the RRT planning technique as a way to initialize pseudospectral methods. Finally, it shows the improvement that the RRT initialization brings to spacecraft reconfiguration problems, which are solved using a Gauss pseudospectral method. 


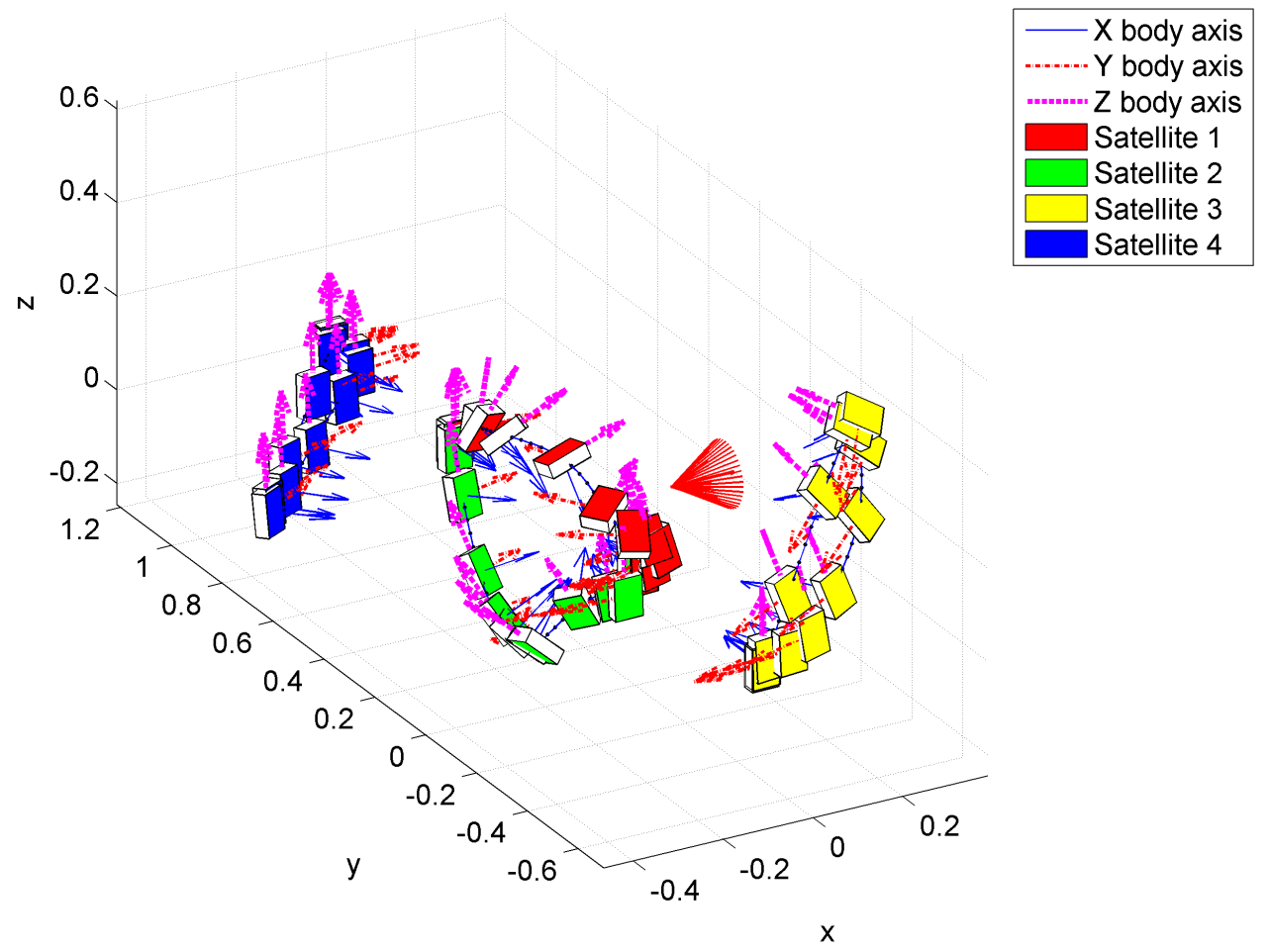

Fig. 9: Final trajectory of the four-spacecraft maneuver with inter-spacecraft and absolute pointing.

\section{Initialization of Pseudospectral Methods}

Pseudospectral methods parameterize the states and controls of a continuous control problem using a basis of global polynomials, and then transcribes the discretized problem into an NLP. The resulting NLP is solved using off-the-shelf nonlinear solvers. Nonlinear solvers typically require a feasible or nearly feasible initial point for their algorithms to converge more robustly and more efficiently to a local optimal solution [58-60]. If this point is not provided by the user, the algorithm usually solves an auxiliary problem to compute it, which can be very time consuming [58].

Therefore, generating a "good" guess to the solver is definitely an important step towards reducing the number of iterations, and consequently the computation time required to solve the NLP. This reduction in computation time will eventually enable real-time path planning onboard spacecraft, thereby reducing future space mission costs and increasing mission quality [61]. It is therefore highly desirable to be able to autonomously solve a path planning problem based on optimal control in real-time. Simplified versions of optimal control problems (e.g., linearized problems) have been already solved in real-time control [27]. However, the main challenge is to implement nonlinear programs in real-time, and get consistent and reliable solutions. 
Pseudospectral methods have the potential to be solved in real-time because they provide highly accurate solutions even with a relatively small number of discretization nodes i.e., a smaller problem size. Algorithms that can determine feasible initial guesses for pseudospectral methods will help reduce the computation time of these methods, and therefore make them more suitable for real-time applications.

\section{Survey of Initialization Techniques}

Note that generating a feasible initial guess for a highly constrained nonlinear problem can be as complicated as solving the nonlinear problem itself. Many researchers have suggested ways to compute acceptable initial guesses, with the general rule of thumb being that the closer the guess is to feasibility, the faster the solver should converge to the optimal solution. There have been different suggestions on how to generate a good guess to initialized a nonlinear solver when solving the transcribed optimal control problem:

1) One common way is that the user develops a good guess for the control using commonengineering-sense [62]. Then the states are computed by using numerical integration. The guess (states and controls) will most likely not be feasible, in the sense that it will not satisfy the boundary conditions. Then looking at the resulting guess and using knowledge of the problem, the user can create a new guess for the control, and repeat the process, until the guess is good enough. But this method can be time consuming, and it is not guaranteed to converge to a feasible answer.

2) Another method is known as the mesh refinement technique [29]. The idea is to start with a coarse grid (i.e., low number of discretization nodes) and use any guess (e.g., randomly chosen) as an initial starting point for the nonlinear solver. Then, if necessary, refine the discretization (i.e., increase the number of discretization nodes), and then repeat the optimization steps using the output of the previous step as the initial guess of the current step. This process can be very time consuming, and therefore current approaches do not appear to be feasible for online planning of complex reconfiguration maneuvers consisting of several spacecraft [29, 58].

3) A third way of initializing the NLP is by using a "warm" start approach [49, 63]. A warm start uses the output of a similar version of the NLP as the initial guess for the actual problem. A similar version is either exactly the same problem run previously (i.e., offline compared to online), or a simplified version of the problem. Warm starts are widely used for active set solvers, but there are still many difficulties in applying them to interior point methods [64]. In addition, using a warm start technique based on an offline computation might not suitable for online implementation where the environment is affected by disturbances, and where previous solutions might not be feasible. So warm start approaches 


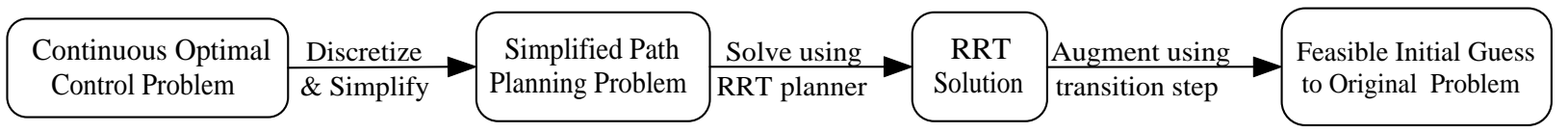

Fig. 10: Steps Involved in Generating a Feasible Initial Guess to the Optimal Control Problem. This guess serves as a starting point to the NLP resulting from the transcription of the optimal control problem using Pseudospectral methods.

should be chosen with care to minimize the inefficiency and inconsistency that they can introduce.

4) A similar approach to warm starts is called homotopy methods that first solve a simpler version of the problem and then continuously modify the solution towards the originally desired problem statement. But homotopy methods require expensive computation and their performance depends on a successful selection of a homotopy which matches best the structure of the problem [65].

The following briefly discusses the RRT-based warm start technique introduced earlier in this paper and then illustrates the significant improvement it provided to the computation time of the pseudospectral method.

\section{RRT Planner Initialization}

The RRT-based initialization has been used in the first stage of the two-stage path planning algorithm described earlier. It is illustrated in Figure 10. It is an efficient warm start approach that initializes the NLP resulting from the transcription of the continuous optimal control problem. This warm start is formulated as an RRT path planning problem, with no differential constraints. The output of the RRT planner is then augmented with feasible dynamics that are propagated from source to destination using an algorithm that ensures feasibility at each node. This process of augmenting the output of the randomized planner is called the transition phase (see section The Augmentation with Feasible Dynamics). The resulting output is a feasible initial guess to the complete optimal control problem. The paper uses an improved version of the bidirectional rapidly-exploring random trees (RRT) that is described in Ref. [22].

\section{Illustration of the RRT improvement}

This section illustrates the improvement that the RRT initialization step brings to optimal control problems solved using pseudospectral methods. To do so, it revisits the multi-spacecraft reconfiguration maneuver problem with path constraints (see Equations (1) - (23)), but places more emphasis on the importance of the role of first step (the RRT initialization) on the solution performance of the optimal control problem formed in the second stage. This initialization 
technique should be straightforward to adapt to several other optimal control problem examples. Recall that the optimal control problem of interest is transcribed into an NLP using the Gauss pseudospectral method (GPM) which has shown promise both in the accuracy of the solution and post-optimality analysis of optimal control problems [26].

To highlight the improvement of the RRT step, several reconfiguration maneuvers of increasing complexity are solved twice: 1) using the RRT step to find a feasible initial guess, and 2) using a "cold" start approach which relies on the nonlinear solver to find an initial guess. The examples consist of reconfiguration maneuvers that include stay outside constraints (e.g., sun avoidance), inter-spacecraft collision avoidance constraints, and obstacle avoidance constraints. These are hard non-convex constraints. But the examples do not include inter-spacecraft constraints such as those described in Figure 1. Including the inter-spacecraft constraints resulted in the nonlinear solver failing to converge when using the cold approach. Thus the RRT initial guess is essential in those cases. Recall that inter-spacecraft constraints are coupling constraints i.e., they affect, in a coupled way, the position and attitudes of the spacecraft. Therefore the feasible set of problems having coupled constraints is usually a very small region, which explains the difficulties of the nonlinear solver. The examples below are solved using GPOCS [56]. Both optimality and feasibility tolerances are set to $10^{-4}$. It was observed by numerical experimentation that decreasing these tolerances further achieve marginal improvement while significantly increasing computation time [44]. The computation times and costs of the following examples are summarized in Table I and Table II.

Single Spacecraft Maneuver: This example is a simple translation from the position $[0,0,0]^{T}$ to $[1,1,1]^{T}$ with a $180^{\circ}$ rotation around the $\mathrm{Z}$ axis. The spacecraft has to avoid pointing its $\mathrm{X}$ axis in the sun direction, which is represented by the vector $\left[\frac{1}{\sqrt{2}}, \frac{1}{\sqrt{2}}, 0\right]^{T}$, surrounded by a cone of $30^{\circ}$ half angle. It also has to avoid colliding with a fixed obstacle centered at $[0.6,0.5,0.5]^{T}$, with radius equal to $0.15 \mathrm{~m}$.

Diagonally Crossing Maneuver: This problem consists of a three-spacecraft maneuver. Spacecraft 1 and 2 start at $[0,0,0]^{T}$ and $[1,1,1]^{T}$, the opposite corners of a cube of side 1 . They must switch position and make a $90^{\circ}$ rotation around the inertial $\mathrm{Z}$ axis. A third spacecraft, spacecraft 3 starts at $[1,0,0]^{T}$ and ends at $[0,1,1]^{T}$. It also performs at $90^{\circ}$ rotation. The maneuver of spacecraft three crosses diagonally those of the other two spacecraft. All three spacecraft have to avoid a fixed obstacle located at $[0.3,0.3,0.3]^{T}$ with radius equal to $0.2 \mathrm{~m}$. They also must avoid pointing their body $X$ axis in the sun direction, which is represented by the vector $\left[\frac{1}{\sqrt{2}}, \frac{1}{\sqrt{2}}, 0\right]^{T}$, surrounded by a cone of $30^{\circ}$ half angle. Figure 11 shows the RRT initial guess, the final trajectory of the problem that uses the RRT guess, and the final trajectory that uses the cold start approach. Two circles surrounding the spacecraft help visualize the actual boundaries of the spacecraft. 


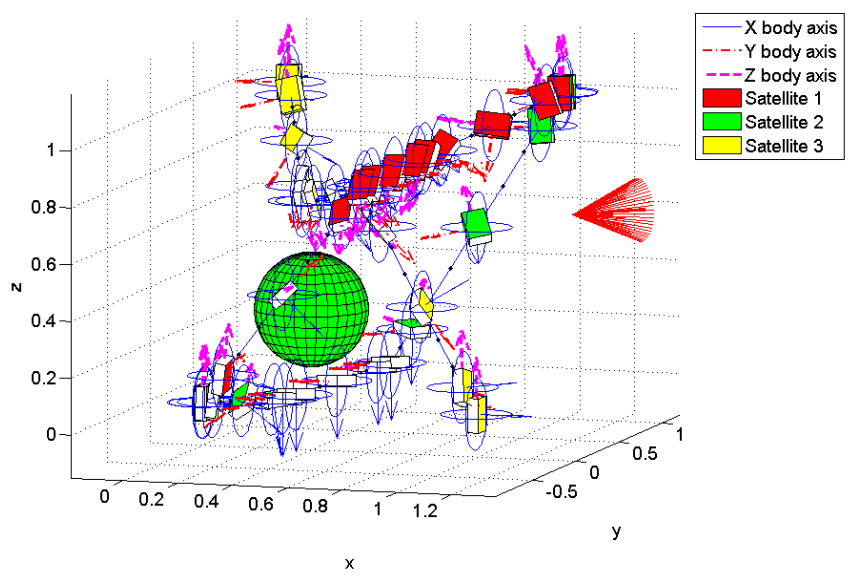

a. RRT Initial Guess

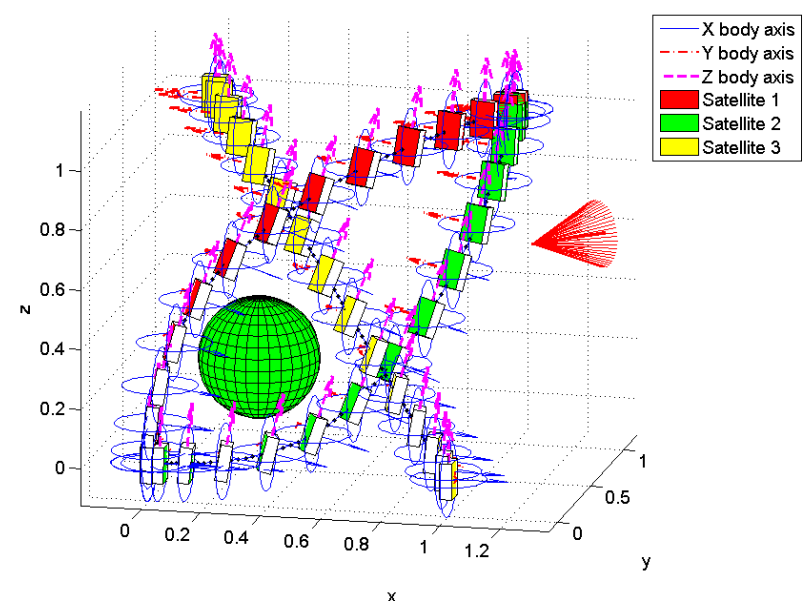

b. Final trajectory solved using RRT initial guess.

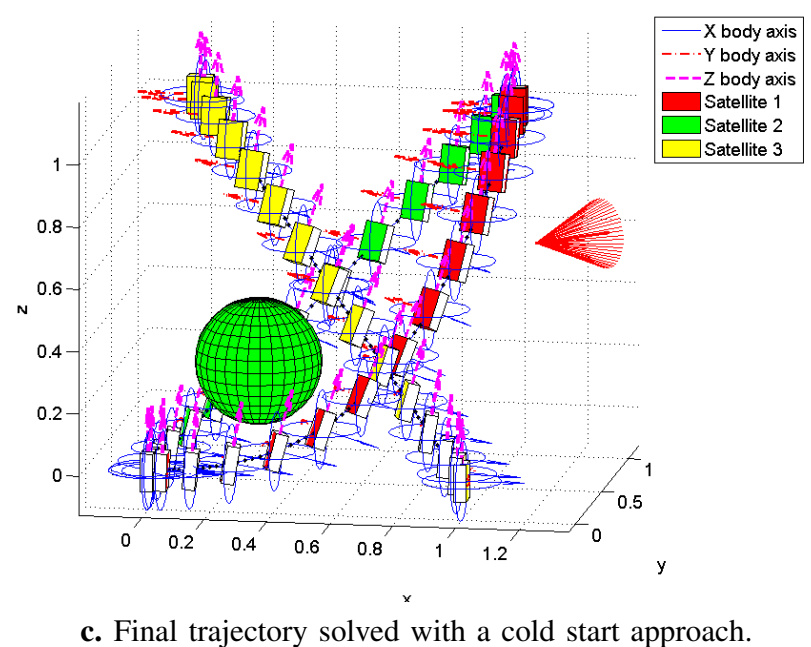

Fig. 11: RRT Path and Final Trajectories for the Three-Spacecraft Example 
TABLE I: Comparison of computation times of reconfiguration maneuvers for formation of increasing size solved using a Gauss pseudospectral method (average over 10 runs)

\begin{tabular}{c||c|c|c}
\hline \multicolumn{1}{c||}{} & \multicolumn{2}{c|}{ Time (s) } & \\
Example & w/o RRT & with RRT & Time Ratio \\
\hline \hline $1 \mathrm{~s} / \mathrm{c}$ & 35 & 24 & 1.46 \\
$2 \mathrm{~s} / \mathrm{c}$ & 103 & 67 & 1.54 \\
$3 \mathrm{~s} / \mathrm{c}$ & 335 & 171 & 1.96 \\
$4 \mathrm{~s} / \mathrm{c}$ & 478 & 228 & 2.10 \\
$5 \mathrm{~s} / \mathrm{c}$ & 834 & 356 & 2.34 \\
\hline
\end{tabular}

Formation Reflection with Four Spacecraft: This example consists of four spacecraft that start in a square formation, and end in another reflected square formation. Spacecraft 1 and spacecraft 3 start at $[0,1,0]^{T}$ and $[2,1,0]^{T}$, respectively. They must end at their original positions. They must also rotate $90^{\circ}$ around the inertial $\mathrm{Z}$ axis. Spacecraft 2 and 4 start at $[1,0,0]^{T}$ and $[1,2,0]^{T}$, and must switch their position. They must also rotate $180^{\circ}$ around the inertial $\mathrm{Z}$ axis. All spacecraft must avoid pointing their $\mathrm{X}$ body axis inside two cones of $50^{\circ}$ along the $\mathrm{X}$ and $-\mathrm{X}$ inertial directions. They must also avoid colliding with a fixed obstacle located at the center of the square, with radius equal to $0.25 \mathrm{~m}$. The pointing constraints lead to a non-trivial rotation maneuvers for both spacecraft 1 and 3. The fixed obstacle makes the trajectories of spacecraft 2 and 4 a more challenging one. In Summary, the maneuver is a reflection of a square formation around a line passing through the fixed positions of Spacecraft 1 and 3.

Formation Rotation with Five Spacecraft: This example consists of five spacecraft starting in a pyramid formation. Spacecraft 5 starts at the apex of the pyramid, while spacecraft 1 through 4 form its square base. Each spacecraft must move to the next spacecraft position in the sequence (spacecraft 2 moves to the spacecraft 1 position, 3 to 2, 4 to 3, 5 to 4, and 1 to 5). Each spacecraft must also end the maneuver pointing in the direction where the next spacecraft in the sequence was pointing at the beginning of the maneuver. Thus, the final configuration is a rotated version of the original pyramid formation. The spacecraft must avoid pointing their $\mathrm{X}$ body axis towards the sun direction represented by the vector $\left[\frac{1}{\sqrt{2}}, \frac{1}{\sqrt{2}}, 0\right]^{T}$, surrounded by a cone of $20^{\circ}$ half angle. They must also avoid colliding with two fixed obstacles of radius equal to $0.15 \mathrm{~m}$. Figure 12 shows the RRT initial guess, the final trajectory of the problem that uses the RRT guess, and the final trajectory that uses the cold start approach.

\section{Performance Comparison}

Table I summarizes the computation times of the examples used to show the improvement of the RRT initialization on problems solved using pseudospectral methods. The with RRT time includes the time of the RRT step, the transition step, and the time required by the GPOCS 


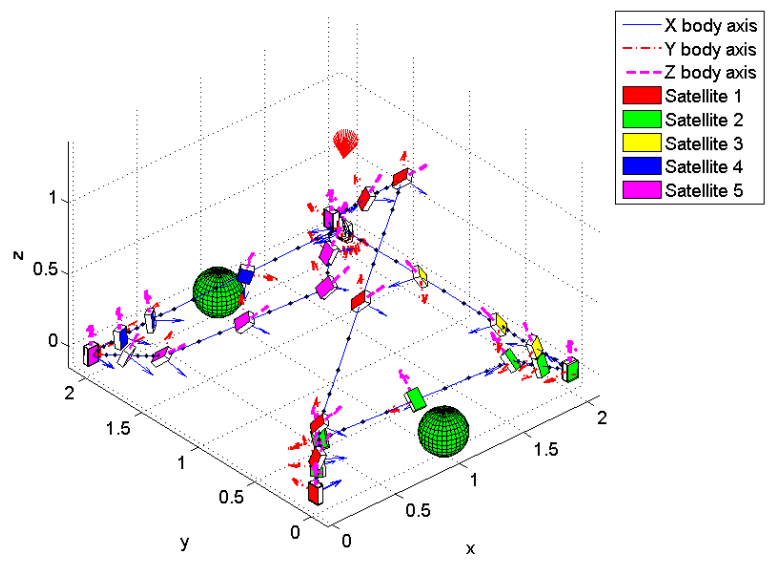

a. RRT Initial Guess

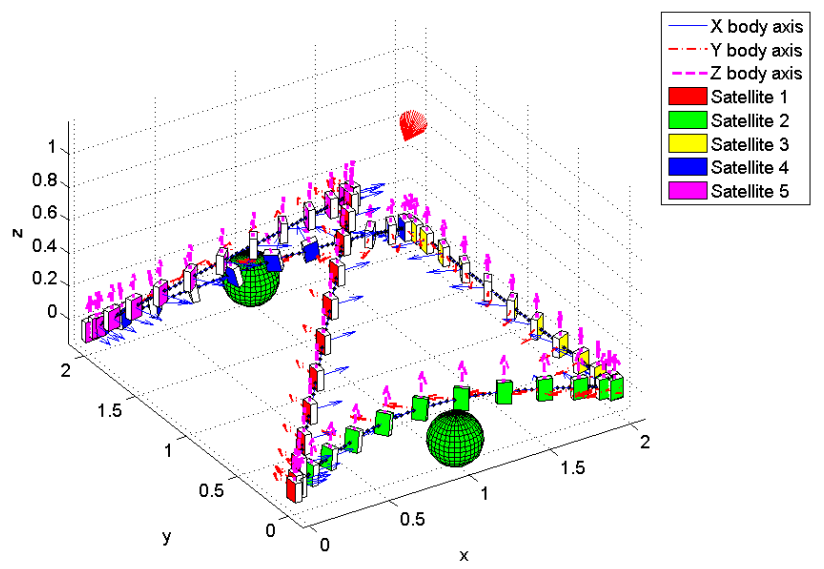

b. Final trajectory solved using RRT initial guess.

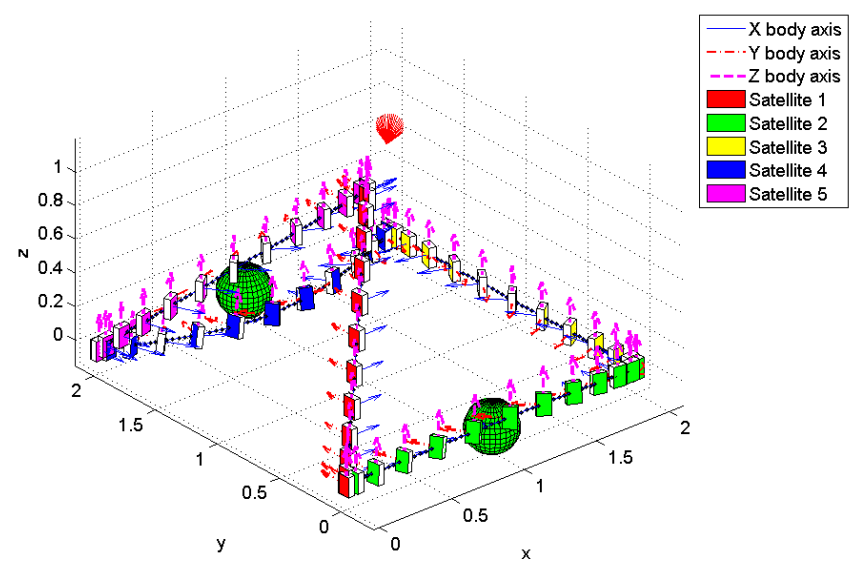

c. Final trajectory solved with a cold start approach.

Fig. 12: RRT Path and Final Trajectories for the Five-Spacecraft Example 


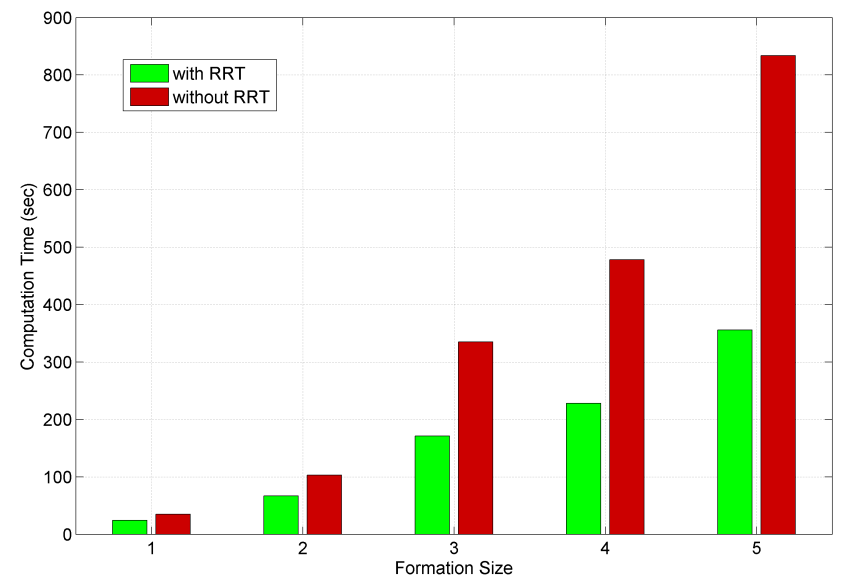

Fig. 13: Comparison of computation times of a series of reconfiguration maneuvers of increasing size solved using a Gauss pseudospectral method (average over 10 runs). The with RRT includes computation times of the RRT planner and the transition step.

TABLE II: Comparison of final costs of reconfiguration maneuvers for formation of increasing size solved using a Gauss pseudospectral method (average over 10 runs)

\begin{tabular}{c||c|c|c}
\hline \multicolumn{1}{c||}{} & \multicolumn{2}{c|}{ Cost } & Cost \\
Example & w/o RRT & with RRT & Comparison \% \\
\hline \hline $1 \mathrm{~s} / \mathrm{c}$ & 0.975 & 1.012 & +3.65 \\
$2 \mathrm{~s} / \mathrm{c}$ & 1.806 & 1.847 & +2.22 \\
$3 \mathrm{~s} / \mathrm{c}$ & 2.752 & 2.821 & +2.45 \\
$4 \mathrm{~s} / \mathrm{c}$ & 4.490 & 4.444 & -1.03 \\
$5 \mathrm{~s} / \mathrm{c}$ & 8.654 & 8.743 & +1.02 \\
\hline
\end{tabular}

to solve the problem. The w/o RRT consists of the time needed by GPOCS to solve the same problems without an initial RRT guess i.e., using a cold start approach. The last column shows the ratio of the w/o RRT times over the with RRT times, i.e., the computation time improvement due to the RRT initial guess.

Figure 13 displays side by side the computation times of both versions of the solutions of the reconfiguration maneuvers as a function of the formation size. The results show that the RRT initialization reduces the GPM [44] computation time, when compared to the w/o RRT case, by a factor increasing from 1.46 to 2.34 with the size of the formation (Figure 13). Clearly, the RRT initial guess improves the scaling of the GPOCS computation time of the solution of the multiple spacecraft reconfiguration problem.

Table II summarizes the final performance values of the same examples. The last column compares the cost of each example solved without an initial RRT guess to the cost of the 
same example solved with an RRT initial guess. A positive value indicates the percentage of improvement (i.e., decrease) in the cost of the w/o RRT case when compared to the with RRT one. Similarly, a negative value indicates the percentage of degradation (i.e., increase) in the cost. The results show that the cost values are very comparable. In the worst case, the cost of $w / o$ RRT version is $3.65 \%$ better. For the largest formation, the five spacecraft reconfiguration shown in Figure 12, the w/o RRT cost is only $1.02 \%$ better.

Finally, it should be emphasized that, when the cold approach is adopted, GPOCS fails (after 10 attempts) to solve examples that include inter-spacecraft pointing constraints. Such examples are described in the illustration of the two-stage approach earlier in this paper. Recall that these constraints tightly couple the position and attitude of the spacecraft. Thus, they significantly increase the complexity of the problem to be solved by the nonlinear solver that GPOCS relies on in its solution process. However, when provided with an RRT guess, GPOCS is able to converge to a solution. So not only does the RRT guess reduce the computation times of the reconfiguration maneuver problem, it also enables the solution of a complex class of reconfiguration problems that include inter-spacecraft constraints.

\section{CONCLUSIONS}

This paper presented a two-stage path planning technique for designing multiple spacecraft reconfiguration maneuvers with various path constraints. The main idea was to combine an improved RRT planner with a Gauss pseudospectral method to obtain highly accurate solutions with reduced computation times that could enable an online implementation. Several complex examples demonstrated the validity of this approach, and it has been used to design reconfiguration maneuvers that were successfully performed on SPHERES micro-satellites onboard the International Space Station [55]. This paper also showed the importance of an initialization step based on an RRT planner to the solution of optimal control problems solved using pseudospectral methods. It showed that this step 1) reduced the computation time of the solutions by up to a factor of 2.34 for a five-spacecraft reconfiguration maneuver, and 2) made it possible to solve a more complex class of such problems that include highly coupled inter-spacecraft pointing constraints.

\section{ACKNOWLEDGMENTS}

This research was funded in part by the Payload Systems Inc. SPHERES Autonomy and Identification Testbed Grant \#012650-001 (PI: Professor David Miller, director of the Space Systems Laboratory at MIT), NASA Grants \#NAG3-2839 and \#NAG5-10440, and Le Fonds Québécois de la Recherche sur la Nature et les Technologies (FQRNT) Graduate Award. The 
authors would like to thank Professor Anil Rao and Dr. Geoffrey Huntington for their technical assistance with the GPOCS software.

\section{REFERENCES}

[1] C. Sultan, S. Seereram, and R. K. Mehra, "Deep Space Formation Flying Spacecraft Path Planning," The International Journal of Robotics Research, vol. 26, no. 4, pp. 405-430, 2007.

[2] P. R. Lawson, "The Terrestrial Planet Finder," in Proceedings of the IEEE Aerospace Conference, vol. 4, March 2001, pp. 2005-2011.

[3] "NASA Laser Interferometer Space Antenna Website." [Online]. Available: http: //lisa.nasa.gov/

[4] C. V. M. Fridlund, "Darwin: The Infrared Space Interferometer Darwin and Astronomy," ESA, Tech. Rep. SP-451, 2000.

[5] A. Das and R. Cobb, "TechSat21Space Missions Using Collaborating Constellations of Satellites," in 12th Annual Small Satellite Conference, no. Paper SSC98-VI-1. Reston, VA: AIAA, August 1998.

[6] "Broad Agency Announcement (BAA07-31) System F6 for Defense Advanced Research Projects Agency (DARPA)," 2007. [Online]. Available: http://www.darpa.mil/ucar/solicit/ baa07-31/f6_baa_final_07-16-07.doc

[7] A. Robertson, G. Inalhan, and J. How, "Spacecraft Formation Flying Control Design for the Orion Mission," in In Proceedings of the AIAA Conference on Navigation, Portland, OR, 1999.

[8] F. H. Bauer, J. O. Bristow, J. R. Carpenter, J. L. Garrison, K. Hartman, T. Lee, A. Long, D. Kelbel, V. Lu, J. P. How, F. Busse, P. Axelrad, , and M. Moreau, "Enabling Spacecraft Formation Flying through Spaceborne GPS and Enhanced Autonomy Technologies," Space Technology, vol. 20, pp. 175-185, 2001.

[9] D. P. Scharf, F. Y. Hadaegh, and S. R. Ploen, "A Survey of Spacecraft Formation Flying Guidance and Control. Part II: Control," in Proceedings of the American Control Conference, vol. 4, 30 June-2 July 2004, pp. 2976-2985.

[10] _ - "A Survey of Spacecraft Formation Flying Guidance and Control. Part I: Guidance," in Proceedings of American Control Conference, vol. 2, Jun 2003, pp. 1733-1739.

[11] I. M. Garcia, "Nonlinear Trajectory Optimization with Path Constraints Applied to Spacecraft Reconfiguration Maneuvers," Master's thesis, Massachussets Institute of Technology, 2005.

[12] A. Richards, T. Schouwenaars, J. P. How, and E. Feron, "Spacecraft Trajectory Planning with Avoidance Constraints," Journal of Guidance control and dynamics, vol. 25, no. 4, pp. 755-764, 2002.

[13] R. K. Prasanth, J. D. Boskovic, and R. K. Mehra, "Mixed integer/LMI programs for lowlevel path planning," in Proceedings of the American Control Conference, vol. 1, 2002, pp. 608-613 vol.1.

[14] F. McQuade, R. Ward, and C. McInnes, "The Autonomous Configuration of Satellite Formations using Generic Potential Functions," in Proceedings of the International Symposium Formation Flying, 2002. 
[15] R. O. Saber, W. B. Dunbar, and R. M. Murray, "Cooperative Control of Multi-Vehicle Systems using Cost Graphs and Optimization," in Proceedings of the American Control Conference, vol. 3, 2003, pp. 2217-2222.

[16] E. Frazzoli, M. A. Dahleh, E. Feron, and R. P. Kornfeld, "A Randomized Attitude Slew Planning Algorithm for Autonomous Spacecraft," AIAA Guidance, Navigation, and Control Conference and Exhibit, Montreal, Canada, 2001.

[17] L. E. Kavraki, P. Svestka, C. L. J, and M. H. Overmars, "Probabilistic Roadmaps for Path Planning in High-Dimensional Configuration Spaces," IEEE Transactions on Robotics and Automation, vol. 12, no. 4, pp. 566-580, 1996.

[18] L. E. Kavraki, M. N. Kolountzakis, and J. C. Latombe, "Analysis of probabilistic roadmaps for path planning," in IEEE International Conference on Robotics and Automation, vol. 4, 1996, pp. 3020-3025 vol.4.

[19] J. M. Phillips, L. E. Kavraki, and N. Bedrosian, "Spacecraft Rendezvous and Docking With Real-Time Randomized Optimization," in AIAA Guidance, Navigation and Control Conference, Austin, TX, August 2003.

[20] E. Frazzoli, "Quasi-Random Algorithms for Real-Time Spacecraft Motion Planning and Coordination," Acta Astronautica, vol. 53, no. 4-10, pp. 485-495, 2003.

[21] I. Garcia and J. P. How, "Trajectory Optimization for Satellite Reconfiguration Maneuvers With Position and Attitude Constraints," in American Control Conference, 2005. Proceedings of the 2005, vol. 2, 2005, pp. 889-894.

[22] — - "Improving the Efficiency of Rapidly-exploring Random Trees Using a Potential Function Planner," in IEEE Conference on Decision and European Control Conference, 2005, pp. 7965-7970.

[23] I. Ross and C. D'Souza, "Rapid Trajectory Optimization of Multi-Agent Hybrid Systems," in AIAA Guidance, Navigation, and Control Conference and Exhibit, 2004.

[24] G. T. Huntington and A. V. Rao, "Optimal Configuration Of Spacecraft Formations Via A Gauss Pseudospectral Method," Advances in the Astronautical Sciences, Part I, vol. 120, pp. 33-50, 2005.

[25] Q. Gong, W. Kang, and I. M. Ross, "A Pseudospectral Method for the Optimal Control of Constrained Feedback Linearizable Systems," in Proceedings of Conference on Control Applications on, 2005, pp. 1033-1038.

[26] G. T. Huntington and A. V. Rao, "Optimal Reconfiguration Of A Tetrahedral Formation Via A Gauss Pseudospectral Method," Advances in the Astronautical Sciences, Part II, vol. 123, pp. 1337-1358, 2006.

[27] G. T. Huntington, "Advancement and Analysis of a Gauss Pseudospectral Transcription for Optimal Control Problems," Ph.D. dissertation, Massachusetts Institute of Technology, June 2007.

[28] J. H. Reif, "Complexity of the Mover's Problem and Generalizations," in IEEE Symposium on Foundations of Computer Science, 1979, pp. 421-427.

[29] J. T. Betts and W. P. Huffman, "Mesh Refinement in Direct Transcription Methods for Optimal Control," Optimal Control Applications and Methods, vol. 19, no. 1, pp. 1-21, 1998.

[30] S. LaValle, "Rapidly-Exploring Random Trees: A New Tool for Path Planning. Technical Report 98-11, Computer Science Dept., Iowa State University,” Oct 1998. 
[31] S. M. LaValle and J. J. J. Kuffner, "Randomized kinodynamic planning," in Robotics and Automation, 1999. Proceedings. 1999 IEEE International Conference on, vol. 1, 1999, pp. 473-479.

[32] D. A. Benson, G. T. Huntington, T. P. Thorvaldsen, and A. V. Rao, "Direct Trajectory Optimization and Costate Estimation via an Orthogonal Collocation Method," Journal of Guidance, Control, and Dynamics, vol. 29, no. 6, pp. 1435-1440, November-December 2006.

[33] B. R. Donald, P. G. X. J. F. Canny, and J. H. Reif, "Kinodynamic Motion Planning," Journal of the ACM, vol. 40, no. 5, pp. 1048-1066, 1993.

[34] J. J. Kuffner, "Motion Planning with Dynamics," Physiqual, March 1998.

[35] D. Hsu, R. Kindel, J. Latombe, and S. Rock, "Randomized Kinodynamic Motion Planning with Moving Obstacles," Int. J. Robotics Research, vol. 21, no. 3, pp. 233-255, 2002.

[36] E. Frazzoli, M. A. Dahleh, and E. Feron, "Real-time Motion Planning for Agile Autonomous Vehicles," in American Control Conference, vol. 1, 2001, pp. 43-49.

[37] I. Belousov, C. Esteves, J. P. Laumond, and E. Ferre, "Motion Planning for the Large Space Manipulators with Complicated Dynamics," in International Conference on Intelligent Robots and Systems, IEEE/RSJ, 2005, pp. 2160-2166.

[38] S. M. Lavalle, Planning Algorithms. Cambridge University Press, 2006.

[39] J. T. Betts, "Survey of Numerical Methods for Trajectory Optimization," Journal of Guidance, Control, and Dynamics, vol. 21, no. 2, pp. 193-207, 1998.

[40] G. Elnagar, M. A. Kazemi, and M. Razzaghi, "The pseudospectral Legendre Method for Discretizing Optimal Control Problems," IEEE Transactions on Automatic Control, vol. 40, no. 10, pp. 1793-1796, 1995.

[41] G. N. Elnagar and M. A. Kazemi, "Pseudospectral Chebyshev Optimal Control of Constrained Nonlinear Dynamical Systems," Comput. Optim. Appl., vol. 11, no. 2, pp. 195-217, 1998.

[42] I. M. Ross and F. Fahroo, "Convergence of Pseudospectral Discretizations of Optimal Control Problems," in Proceedings of the 40th IEEE Conference on Decision and Control, vol. 4, 2001, pp. 3175-3177.

[43] M. D. Shuster, "Survey of Attitude Representations," Journal of the Astronautical Sciences, vol. 41, pp. 439-517, Oct. 1993.

[44] G. S. Aoude, "Two-Stage Path Planning Approach for Designing Multiple Spacecraft Reconfiguration Maneuvers and Application to SPHERES onboard ISS," Master's thesis, Massachusetts Institute of Technology, September 2007. [Online]. Available: http://dspace.mit.edu/bitstream/handle/1721.1/42050/230816006.pdf?sequence $=1$

[45] D. P. Scharf, F. Y. Hadaegh, and B. H. Kang, "On the validity of the double integrator approximation in deep space formation flying," in International Symposium Formation Flying Missions \& Technologies, Toulouse, France, October 2002.

[46] A. G. Richards, "Trajectory Optimization using Mixed-Integer Linear Programming," Master's thesis, Massachusetts Institute of Technology, June 2002.

[47] Y. Kim, M. Mesbah, and F. Y. Hadaegh, "Dual-spacecraft formation flying in deep space - Optimal collision-free reconfigurations," Journal of Guidance, Control, and Dynamics, vol. 26, no. 2, pp. 375-379, March 2003.

[48] A. Rahmani, M. Mesbahi, and F. Y. Hadaegh, "On the Optimal Balanced-Energy Formation 
Flying Maneuvers," 2005 AIAA Guidance, Navigation, and Control Conference and Exhibit; San Francisco, CA; USA; 15-18 Aug. 2005, pp. 1-8, 2005.

[49] P. E. Gill, W. Murray, and M. A. Saunders, "User's Guide For SNOPT 5.3: A Fortran Package For Large-Scale Nonlinear Programming," 1999.

[50] L. N. Trefethen, Spectral Methods in Matlab. Philadelphia: SIAM Press, 2000.

[51] D. Benson, "A Gauss Pseudospectreal Transcription for Optimal Control," Ph.D. dissertation, Massachusetts Institute of Technology, 2005.

[52] D. E. Kirk, Optimal Control Theory: An Introduction. Dover Publications, 1970.

[53] A. S. Otero, A. Chen, D. W. Miller, and M. Hilstad, "SPHERES: Development of an ISS Laboratory for Formation Flight and Docking Research," in IEEE Aerospace Conference Proceedings, vol. 1, 2002, pp. 59-73.

[54] J. Enright, M. Hilstad, A. Saenz-Otero, and D. Miller, "The SPHERES Guest Scientist Program: Collaborative Science on the ISS," in Proceedings of the IEEE Aerospace Conference, vol. 1, 2004.

[55] G. S. Aoude, J. P. How, and D. W. Miller, "Reconfiguration Maneuver Experiments using the SPHERES tesbed onboard the ISS," in 3rd International Symposium on Formation Flying, Missions and Technologies. Noordwijk, The Netherlands: ESA/ESTEC, April 2008.

[56] A. V. Rao, User's Manual for GPOCS Version 1.1: A MATLAB Implementation of the Gauss Pseudospectral Method for Solving Non-Sequential Multiple-Phase Optimal Control Problems, report 1 beta ed., May 2007.

[57] D. Hsu, L. Kavraki, J. Latombe, R. Motwani, and S. Sorkin, "On finding narrow passages with probabilistic roadmap planners," in Proceedings of the third workshop on the algorithmic foundations of robotics on Robotics, Houston, TX, 1998, pp. 141-153.

[58] J. T. Betts, Practical methods for optimal control using nonlinear programming, ser. Advances in Design and Control. Philadelphia, PA: Society for Industrial and Applied Mathematics (SIAM), 2001, vol. 3.

[59] J. W. Chinneck, "The constraint consensus method for finding approximately feasible points in nonlinear programs," INFORMS Journal on Computing, vol. 16, no. 3, pp. 255-265, 2004.

[60] O. A. Elwakeil and J. S. Arora, "Methods for finding feasible points in constrained optimization," AIAA journal, vol. 33, no. 9, pp. 1715-1719, 1995.

[61] N. Muscettola, B. Smith, S. Chien, C. Fry, G. Rabideau, K. Rajan, and D. Yan, "Onboard Planning for Autonomous Spacecraft," in In Proceedings of the Fourth International Symposium on Artificial Intelligence, Robotics, and Automation for Space (iSAIRAS), July 1997.

[62] I. M. Ross, User's Manual for DIDO: A MATLAB Application Package for Solving Optimal Control Problems, Monterey, California, February 2004.

[63] J. Mitchell and B. Borchers, "Solving Real-World Linear Ordering Problems Using a Primal-Dual Interior Point Cutting Plane Method," Annals of Operations Research, vol. 62, no. 1, pp. 253-276, Dec. 1996.

[64] R. A. Bartlett, A. Wachter, and L. T. Biegler, "Active Set vs. Interior Point Strategies for Model Predictive Control," in Proceedings of the American Control Conference, vol. 6, 2000, pp. 4229-4233. 
[65] K. Reif, K. Weinzierl, A. Zell, and R. Unbehauen, "Application of homotopy methods to nonlinear control problems," Decision and Control, 1996., Proceedings of the 35th IEEE, vol. 1, pp. 533-538 vol.1, 11-13 Dec 1996. 